Copyright by the American Physical Society. Paul, M. R. ; Clark, M. T. ; Cross, M. C., Oct 24, 2013. microscale and nanoscale elastic objects in a viscous fluid," PHYSICAL REVIEW E 88(4): 043012. DOI: 10.1103/

PHYSICAL REVIEW E 88, 043012 (2013)

\title{
Coupled motion of microscale and nanoscale elastic objects in a viscous fluid
}

\author{
M. R. Paul and M. T. Clark \\ Department of Mechanical Engineering, Virginia Tech, Blacksburg, Virginia 24061, USA \\ M. C. Cross \\ Department of Physics, California Institute of Technology, Pasadena, California 91101, USA
}

(Received 11 July 2013; published 24 October 2013)

\begin{abstract}
We study the coupled dynamics of two closely spaced micron or nanoscale elastic objects immersed in a viscous fluid. The dynamics of the elastic objects are coupled through the motion of the surrounding viscous fluid. We consider two cases: (i) one object is driven externally by an imposed harmonic actuation force and the second object is passive and (ii) both objects are driven by a Brownian force to yield stochastic dynamics. Using a harmonic oscillator approximation for the elastic objects and the unsteady Stokes equations to describe the fluid dynamics, we develop analytical expressions for the amplitude and phase of the displacement of the oscillating objects. For the case of an imposed actuation we use an impulse in force to determine the resulting dynamics over all frequencies. For the Brownian-driven objects the stochastic dynamics are found using the fluctuation-dissipation theorem. We validate our theoretical expressions by comparison with results from finite-element numerical simulations of the complete fluid-solid interaction problem. Our results yield interesting features in the amplitude and phase of the displacement of the elastic objects due to the fluid motion. We find that the dynamics depend on the separation of the objects, a measure of the mass loading due to the fluid, and the frequency parameter which acts as a frequency-based Reynolds number. Our results are valid over the range of parameters typical of micron and nanoscale elastic objects in fluid. The range of dynamics found can be understood in terms of the interplay between the viscous and potential components of the fluid flow field described by the unsteady Stokes equation for an oscillating cylinder. For small values of the frequency parameter, typical of nanoscale elastic objects, the dynamics are overdamped due to the dominance of viscous forces over inertial forces. For moderate and large values of the frequency parameter, typical of micron-scale elastic objects, we find that the dynamics of the fluid-coupled objects exhibits an interesting mode splitting to yield a bimodal signature in the amplitude-frequency plots. We find that the mode splitting can be described using a normal mode analysis containing only potential fluid interactions between the cylinders.
\end{abstract}

DOI: 10.1103/PhysRevE.88.043012

PACS number(s): 47.61.-k, 47.63.mf, 05.40.-a, 83.10.Mj

\section{INTRODUCTION}

The rapid advance of micro- and nanoscale technologies has made possible the measurement of fluid properties with unprecedented precision by using the high-frequency dynamics of elastic objects in viscous fluid [1,2]. For example, the dynamics of micron-scale cantilevers in fluid are at the heart of atomic force microscopy techniques for use in liquids on biological samples (cf. Refs. [3,4]). Interpreting the stochastic motion of micron-scale beads in fluid is commonly used in microrheology [2]. The desire for better time resolution and improved force sensitivity has contributed to the push toward the nanoscale and improved capabilities [5,6]. Significant improvements to performance, and new capabilities, are possible by using large arrays of elastic objects in fluid [5]. For example, consider an array of many oscillating elastic objects in fluid where each individual object is tailored to probe a different quantity of interest such as the cantilever-based electronic nose proposed by Battiston et al. [7]. The desire to build such devices on a single chip will require the oscillating objects to be in close proximity. It is well known that the fluid motion induced by an oscillating object at small Reynolds numbers is long range compared to a measure of the object's characteristic size. As a result of the long-range fluid motion the dynamics of arrays of elastic objects will be coupled. The coordinated motion of arrays of structures is a common situation in biological systems, such as the synchronized motion of cilia [8] and flagella [9], which play important roles in many biological processes.

In some situations of interest it is desirable to minimize the fluid coupling to maintain the independent motion of the objects in the array. A particular example of interest would be the development of a single molecule biodetector that relies on the correlated motion of two closely spaced elastic objects with a molecule of interest tethered between them. This approach has been successfully applied by Meiners and Quake [10] to probe the dynamics of a DNA molecule tethered between two micron-scale beads held in optical traps. It has been suggested to extend these ideas to micron and nanoscale cantilevers [5]. In these cases, an understanding of the fluid dynamics due to the motion of the elastic objects is essential in order to extract the correlations due to the tethered molecule of interest.

However, in other situations of interest the fluid-coupled motion of the objects is of direct interest by itself. For example, in two-point microrheology, an understanding of the correlated motion of two micron-scale beads has led to important new insights regarding the rheology of complex fluids [2]. The correlated motion of two closely spaced atomic force microscope cantilevers has also been used to explore the rheology of fluids [11]. In biological systems the hydrodynamic interactions between flagella facilitate their synchronization which enhances motility of microorganisms [12].

In this paper we focus on cases where the fluid motion is a result of the dynamics of the objects. We will consider 
that the objects can be driven by an external actuation force or by a Brownian force. Commonly used approaches for the external actuation of elastic objects are piezoelectric [3], magnetomotive [13], thermoelastic [14], and acoustic [15]. The stochastic motion of Brownian-driven objects is due to the inherent thermal motion of matter at a finite temperature. An additional advantage of measuring the correlated dynamics of a pair of objects driven by Brownian motion is a significant improvement in force sensitivity. This is because the Brownian force on each object is independent and does not contribute to their correlations. The magnitude of displacement of the correlated motion is on an order of magnitude smaller (for typical values of the separation of the two elastic objects) than the displacement of a single elastic object due to the Brownian force. For example, this has been exploited in the development of femtonewton force spectroscopy using micron-scale beads in fluid [10] and in the development of extremely sensitive gravitational wave detectors [16].

Meiners and Quake provide a theoretical description of the correlated motion of micron-scale beads in fluid that are held in optical traps [10]. In this case, the amplitude and frequency of the bead motion is sufficiently small such that inertial forces can be completely neglected and the fluid motion can be described by the Stokes equation [17]. However, as technology pushes towards the use of micron and nanoscale silicon-based elastic objects, such as beams or nanowires, the high-frequency motion makes inertia significant. As a result, the fluid motion must be described by the unsteady Stokes equation which, despite its linearity, is considerably more complex. An analytical solution for the coupled motion of a pair of elastic objects in fluid at these conditions is not available and is a major contribution of this paper.

There has been progress in building our physical understanding of the fluid-coupled dynamics of elastic objects in fluid using numerical approaches. The correlated motion of an array of cantilevers in a viscous fluid that are driven by an external actuation has been explored by Basak and Raman [18] using highly efficient boundary integral methods that exploit the linearity of the underlying equations. In this work, each cantilever in the array is driven independently and a numerical solution for the fluid motion is obtained. The fluid motion is used to analyze the pressure and viscous forces acting on the cantilevers and the variation of these forces with the frequency of oscillation and cantilever spacing in the array was explored. The elastic nature of the cantilevers was not considered. This yielded physical insights into the force interactions due to the fluid on an array of driven oscillators [18]. Our work, presented here, differs in that we solve the complete fluid-solid interaction problem to yield the coupled dynamics of the array that results from their fluid interactions.

The stochastic motion of a pair of nanoscale cantilevers in a viscous fluid has been explored where the stochastic dynamics are computed using the fluctuation-dissipation theorem $[19,20]$. This work has been extended to include a pair of micron-scale cantilevers [21]. Recent experimental measurements of the correlated motion of a pair of micronscale cantilevers in several Newtonian fluids show very good agreement with the theoretical predictions [22]. The numerical investigations of the stochastic dynamics of small cantilever pairs in fluid have yielded several important physical insights.
The dynamics of nanoscale cantilevers in a viscous fluid are overdamped due to the dominance of viscous forces over inertial forces. This yields noise spectra that are very broad with a significant reduction in the oscillator's quality factor and resonant frequency in fluid. The cross-correlations in the cantilever displacement exhibit a bimodal signature with regions of positive and negative correlations. This is in striking contrast to the purely anticorrelated signature of the crosscorrelations in bead position found when the effects of inertia could be completely neglected [10]. The dynamics of a pair of micron-scale cantilevers yielded higher-quality factors, less reduction of the resonant frequency in fluid, and multimodal features in the cross-correlations in cantilever displacement. It was suggested that these features of the correlated dynamics of oscillators in fluid could be described by the interplay between the viscous and potential components of the flow field described by the unsteady Stokes equation [19].

In this paper we present an analytical treatment of the fluid-coupled motion of a pair of elastic objects oscillating at high frequency with small amplitude and in a viscous fluid. Our analysis is done using the flow field given by the unsteady Stokes equation for oscillating cylinders in fluid. The remainder of the paper is organized as follows. In Sec. II we discuss the flow field caused by an oscillating cylinder in a viscous fluid. In Sec. III we provide the analytical expressions for the fluid force acting on an oscillating cylinder. We initially develop the expressions using a reference frame that is stationary with respect to a quiescent background flow field. We then extend this analysis to the case of an oscillating cylinder placed in an oscillating background flow field. In this analysis it will be convenient to use a noninertial reference frame attached to the oscillating background flow field. In Sec. IV we use these results to describe the fluid-coupled motion of a pair of cylinders. In Sec. V we compare our theoretical predictions with finite-element numerical simulation and then use the theoretical expressions to explore the dynamics of a pair of cylinders for a broad range of conditions. Finally, in Sec. VI we provide some concluding remarks.

\section{THE FLOW FIELD CAUSED BY AN OSCILLATING CYLINDER IN A QUIESCENT FLUID}

The fluid motion resulting from a single oscillating cylinder of radius $a$ for low-Reynolds-number flow was originally solved by Stokes [23] (cf. Refs. [24,25]). We follow the discussion of Rosenhead [24] and introduce only the essential results that will be used in our investigation of the correlated motion of a pair of elastic objects coupled by the fluid motion.

We first consider the one-dimensional motion of a cylinder that is undergoing periodic oscillations with a velocity

$$
\vec{u}_{1}=U_{1} e^{i \omega t} \vec{e}_{x}=A_{1} \omega e^{i \omega t} \vec{e}_{x},
$$

where $U_{1}$ is the magnitude of the velocity, $A_{1}$ is the amplitude of displacement, $\omega$ is the radial frequency of oscillation, $\vec{e}_{x}$ is a unit vector in the direction of oscillation, $i$ is the imaginary unit $i=\sqrt{-1}$, and the cylinder displacement is $\vec{x}_{1}=-i A_{1} e^{i \omega t} \hat{e}_{x}$.

In this case, the reference frame is stationary and the cylinder is oscillating. A schematic is shown in Fig. 1 illustrating the geometry and the conventions used in our choice of coordinates. The equations describing the incompressible 


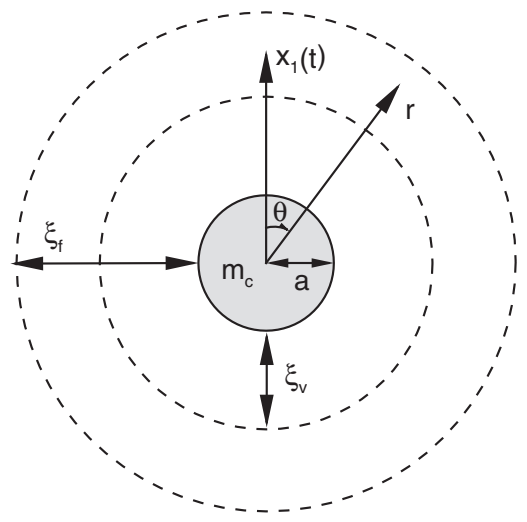

FIG. 1. An oscillating cylinder of radius $a$ and mass $m_{c}$ immersed in a viscous fluid with time-varying displacement $x_{1}(t)$. The conventions used for radial $r$ and azimuthal $\theta$ coordinates are as shown. The region over which viscous effects are significant is $\xi_{v}$ and the region over which there is significant fluid motion is $\xi_{f}$. Both $\xi_{v}(\omega)$ and $\xi_{f}(\omega)$ become larger as the frequency of oscillation is reduced where $\xi_{f}(\omega)>\xi_{v}(\omega)$.

fluid motion are

$$
\begin{aligned}
\rho\left(\frac{\partial \vec{u}}{\partial t}+\vec{u} \cdot \vec{\nabla} \vec{u}\right) & =-\vec{\nabla} p+\mu \nabla^{2} \vec{u}, \\
\vec{\nabla} \cdot \vec{u} & =0,
\end{aligned}
$$

where $\vec{u}$ is the fluid velocity, $p$ is the pressure, $t$ is the time, $\rho$ is the density of the fluid, and $\mu$ is the dynamic viscosity. Although we will find it useful to proceed with the equations in dimensional form, there are two important nondimensional parameters that will be important in our description of the problem. These are the frequency parameter

$$
\beta=\frac{\omega a^{2}}{v},
$$

which expresses the ratio between inertial forces and viscous forces, where $v$ is the kinematic viscosity, and the Reynolds number,

$$
\operatorname{Re}=\frac{U_{1} a}{v}
$$

where the characteristic velocity is $U_{1}=A_{1} \omega$. It is typical in micron and nanoscale applications for the elastic objects to undergo small amplitude $A_{1} \ll 1$ and high-frequency $\omega \gg 1$ oscillations. This yields $\beta \sim 1$ and $\operatorname{Re} \ll 1$ and allows one to neglect the convective nonlinearity in Eq. (2) to give the unsteady Stokes equations,

$$
\begin{gathered}
\rho \frac{\partial \vec{u}}{\partial t}=-\vec{\nabla} p+\mu \nabla^{2} \vec{u}, \\
\vec{\nabla} \cdot \vec{u}=0 .
\end{gathered}
$$

The appropriate boundary conditions are

$$
\begin{gathered}
u_{r}(r \rightarrow \infty, t)=0, \\
u_{\theta}(r \rightarrow \infty, t)=0, \\
u_{r}(r=a, t)=U_{1} \cos \theta, \\
u_{\theta}(r=a, t)=-U_{1} \sin \theta,
\end{gathered}
$$

where $u_{r}$ is the fluid velocity in the radial direction, $u_{\theta}$ is the fluid velocity in the azimuthal direction, and the latter two boundary conditions are the no-slip condition applied to the cylinder surface. The analytical solution to Eqs. (6) and (7) with the boundary conditions given by Eqs. (8)-(11) is

$$
\begin{gathered}
u_{r}=U_{1}\left(\frac{A}{r^{2}}+\frac{B}{r} K_{1}\left[\frac{r}{a} \sqrt{i \beta}\right]\right) e^{i \omega t} \cos \theta, \\
u_{\theta}=U_{1}\left(\frac{A}{r^{2}}-\frac{B}{a} \sqrt{i \beta} K_{1}^{\prime}\left[\frac{r}{a} \sqrt{i \beta}\right]\right) e^{i \omega t} \sin \theta,
\end{gathered}
$$

where the constants $A$ and $B$ are

$$
\begin{gathered}
A=a^{2}\left(1-\frac{2 K_{1}[\sqrt{i \beta}]}{K_{1}[\sqrt{i \beta}]+\sqrt{i \beta} K_{1}^{\prime}[\sqrt{i \beta}]}\right), \\
B=\frac{2 a}{K_{1}[\sqrt{i \beta}]+\sqrt{i \beta} K_{1}^{\prime}[\sqrt{i \beta}]},
\end{gathered}
$$

where $K_{1}$ is the modified Bessel function and $K_{1}^{\prime}$ is its derivative. The flow field around the oscillating cylinder is shown in Fig. 2(a) as contours of the stream function

$$
\psi(r, \theta, t)=U_{1}\left(\frac{A}{r}+B K_{1}\left[\frac{r}{a} \sqrt{i \beta}\right]\right) e^{i \omega t} \sin \theta,
$$
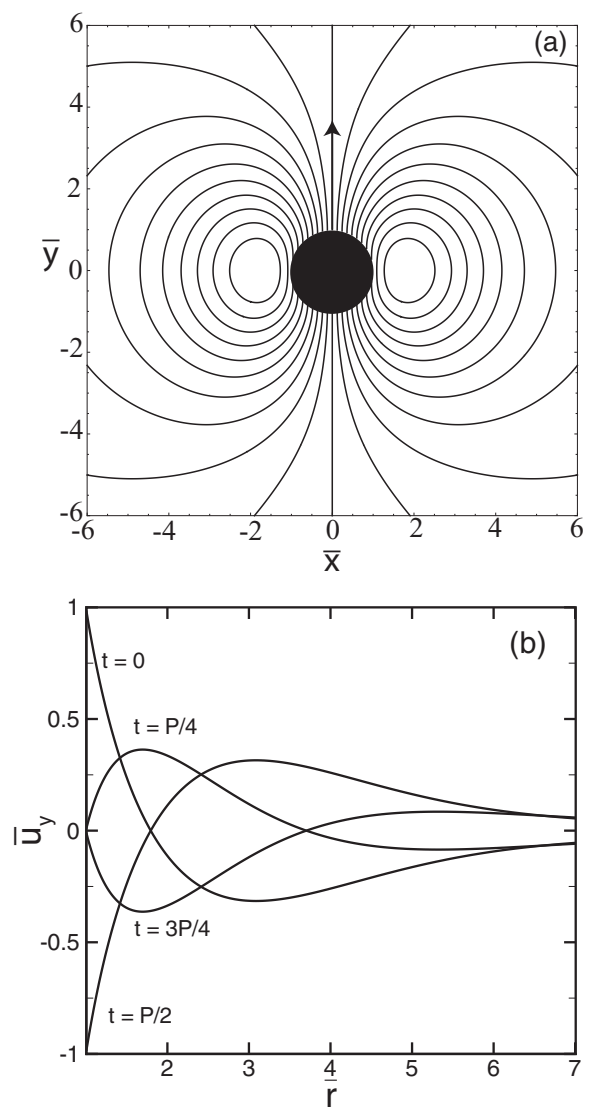

FIG. 2. The flow field resulting from an oscillating cylinder in a viscous fluid for $\beta=1.26$. (a) Contours of the normalized stream function $\bar{\psi}=\psi /\left(U_{1} a\right)$ at time $t=0$ as a function of the normalized Cartesian coordinates $\bar{x}=x / a$ and $\bar{y}=y / a$. The cylinder motion is indicated by the arrow. (b) The variation of the normalized fluid velocity $\bar{u}_{y}=u_{y} / U_{1}$, where $u_{y}$ is given by Eq. (18) with radial distance $\bar{r}=r / a$ and time $t$. The four curves are at different instances of time during a period of oscillation where $P$ is the period. 
where $u_{r}=r^{-1} \partial \psi / \partial \theta$ and $u_{\theta}=-\partial \psi / \partial r$. For example, a cylinder with radius $a=1 \mu \mathrm{m}$ oscillating at $200 \mathrm{KHz}$ in water corresponds to a frequency parameter of $\beta=1$.26. In Fig. 2(a) the normalized stream function $\bar{\psi}=\psi /\left(U_{1} a\right)$ is shown for this case as a function of the normalized radial coordinate $\bar{r}=r / a$ at time $t=0$. At $t=0$ the cylinder is traveling upwards as it crosses the origin with its maximum velocity. In Fig. 2(a) we have used a Cartesian reference frame given by $(\bar{x}=x / a, \bar{y}=$ $y / a)$.

The radial variation of the fluid velocity at $y=0$ is of particular interest for our analysis since this is where the adjacent elastic object will be placed. We will refer to this fluid velocity as $u_{y}(r, t)$, where

$$
\begin{gathered}
u_{y}(r, t)=-u_{\theta}(r, \theta=\pi / 2, t) \\
=-U_{1}\left(\frac{A}{r^{2}}-\frac{B}{a} \sqrt{i \beta} K_{1}^{\prime}\left[\frac{r}{a} \sqrt{i \beta}\right]\right) e^{i \omega t} .
\end{gathered}
$$

The negative sign is due to the convention used for positive $\theta$ as shown in Fig. 1. The variation of $u_{y}$ with time is shown in Fig. 2(b) for $\beta=1.26$. The four curves illustrate the flow field at different instances of time during a single period of oscillation $P$, where $P=2 \pi / \omega$.

It will be insightful to decompose the flow field given by Eqs. (12) and (13) into viscous and potential contributions as

$$
u_{y}(r, t)=u_{p}(r, t)+u_{v}(r, t),
$$

where $u_{p}$ is the potential component and $u_{v}$ is the viscous component given by

$$
\begin{gathered}
u_{p}(r, t)=-U_{1} \frac{A}{r^{2}} e^{i \omega t} \\
u_{v}(r, t)=U_{1} \frac{B}{a} \sqrt{i \beta} K_{1}^{\prime}\left[\frac{r}{a} \sqrt{i \beta}\right] e^{i \omega t} .
\end{gathered}
$$

The potential component exhibits a simple $r^{-2}$ decay in velocity magnitude with increasing radial distance and depends only on the instantaneous velocity of the cylinder. The nonpotential or viscous contribution depends on the previous history of the cylinder motion through the diffusion of momentum with diffusion coefficient $v$. The radial variation of the magnitude of the fluid velocity $\left|u_{y}\right|$ is shown in Fig. 3 as the solid line at time $t=0$. The magnitude of the velocity is scaled by the maximum velocity of the cylinder $U_{1}$ and the radial direction is scaled by the cylinder radius $a$. The dash-dotted line is the variation of the potential component $u_{p}$ and the dashed line is the viscous component $u_{v}$. The potential component of the velocity is opposite in sign to the viscous component and their sum equals the full velocity field.

The viscous contribution is larger than the potential contribution for small radial distances $\bar{r} \lesssim 3$, whereas for $\bar{r} \gtrsim 3$ the viscous component decays rapidly with the potential contribution dominating. As a result, near the cylinder both the viscous and potential contributions are significant, whereas for large distances the potential component dominates. The potential and nonpotential contributions exhibit distinctly different dynamics in terms of both their magnitude and phase relative to the motion of the cylinder.

To quantify the variation of the magnitude, we define length scales over which these two contributions are significant. The length scale $\xi_{v}$ is the distance away from the cylinder over

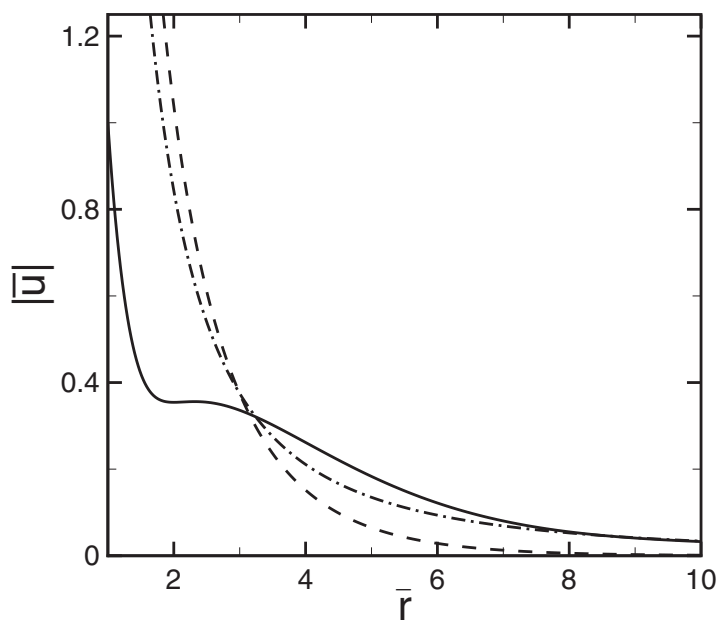

FIG. 3. The radial variation of the magnitude of the fluid velocity evaluated at time $t=0$ for an oscillating cylinder in fluid where $\beta=$ 1.26. The solid line is the fluid velocity $\left|\bar{u}_{y}\right|=\left|u_{y} / U_{1}\right|$, the dashed line is the viscous component $\left|\bar{u}_{v}\right|=\left|u_{v} / U_{1}\right|$, and the dash-dotted line is the potential component $\left|\bar{u}_{p}\right|=\left|u_{p} / U_{1}\right|$. The radial coordinate is normalized by the cylinder radius $\bar{r}=r / a$.

which the viscous contribution is significant. The value of $\xi_{v}$ is the distance away from the cylinder where the viscous component of the velocity field has decayed to $1 \%$ of the maximum value of the fluid velocity when evaluated at $t=0$,

$$
u_{v}\left(r=\xi_{v}, t=0\right)=0.01 U_{1}
$$

The length scale for which there is significant fluid motion is $\xi_{f}$ and is defined using the same convention to be

$$
u_{p}\left(r=\xi_{f}, t=0\right)=0.01 U_{1} .
$$

We note that for $r>\xi_{v}$ the fluid motion is dominated by the potential contribution and $\xi_{f}$ could be determined using $u_{y}$ directly.

In Fig. 4(a) the variation of the two length scales $\xi_{v}$ and $\xi_{f}$ with the frequency parameter $\beta$ are shown. The length scales are normalized by the cylinder radius. As expected, these length scales become quite large for small frequencies of oscillation. In Fig. 4(b) the length scales are normalized by the Stokes length where $\delta_{s}=\sqrt{v / \omega}$. When shown this way it is clear that for over 4 orders of magnitude of the frequency parameter the normalized viscous length scale is of order 10 . This provides a useful heuristic to determine the approximate length scale over which viscous effects are important.

There are significant differences between the phase of the fluid motion at some distance $r$ and the phase of the velocity of the cylinder. To quantify these phase differences we consider the velocity of the cylinder to be

$$
u_{\text {cyl }}(t)=\operatorname{Re}\left(u_{1}\right)=U_{1} \cos (\omega t),
$$

where Re is the real part and $U_{1}$ is the magnitude of the cylinder velocity. Similarly, the radial variation of the fluid velocity, and its decomposition into potential and viscous components, can be expressed using the real part of $u_{y}$ to become

$$
\begin{aligned}
& u_{y}(r, t)=U_{y}(r) \cos \left(\omega t+\phi_{y}(r)\right), \\
& u_{p}(r, t)=U_{p}(r) \cos \left(\omega t+\phi_{p}(r)\right),
\end{aligned}
$$



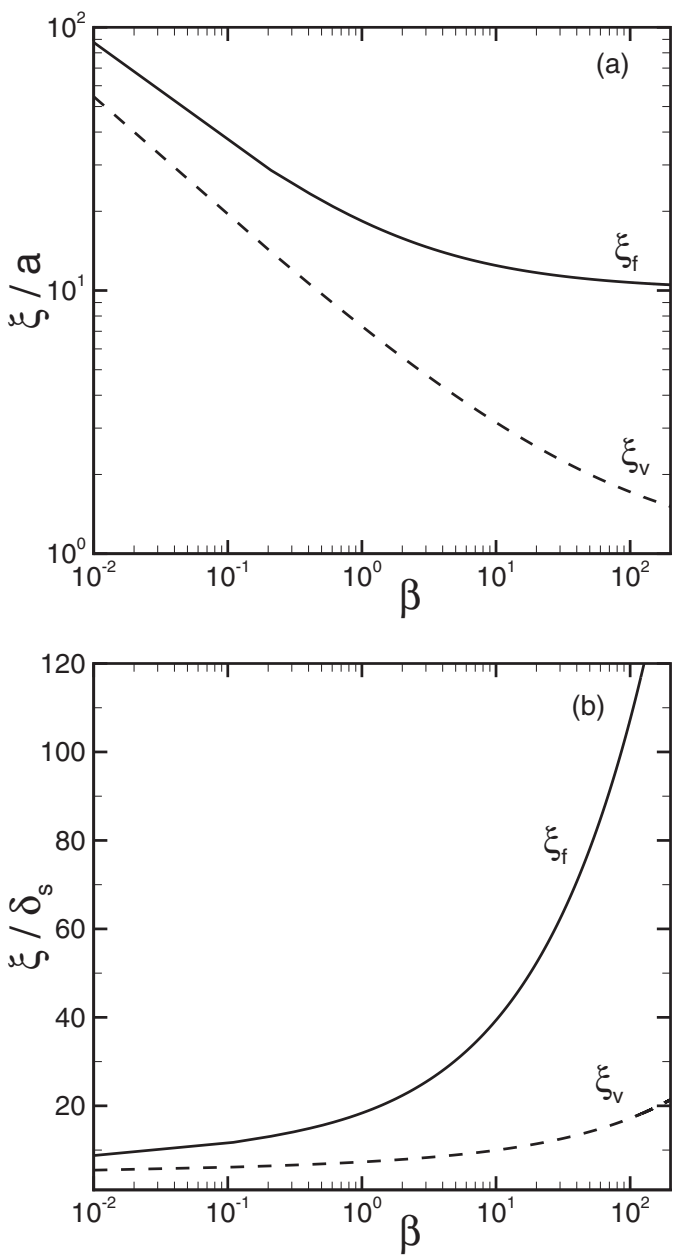

FIG. 4. The variation of the fluid length scales $\xi$ with frequency parameter $\beta . \xi_{v}$ is the length scale over which viscous effects are important (dashed lines) and $\xi_{f}$ is the length scale over which there is significant fluid motion (solid lines). (a) The length scales are normalized using the cylinder radius $a$. (b) The length scales are normalized using the Stokes length $\delta_{s}=\sqrt{v / \omega}$.

$$
u_{v}(r, t)=U_{v}(r) \cos \left(\omega t+\phi_{v}(r)\right)
$$

where $\phi(r)$ is the position dependent relative phase difference between the fluid velocity and the velocity of the cylinder. The velocity $U(r)$ is the position dependent magnitude of the fluid velocity.

These phase differences have direct relevance to the fluidcoupled motion of adjacent elastic objects placed in the fluid. The spatial variation of the different velocity components is shown in Fig. 5(a) at time $t=0$ for $\beta=1.26$. The solid line is the normalized fluid velocity $\bar{u}_{y}=u_{y} / U_{1}$, the dash-dotted line is the normalized potential component $\bar{u}_{p}=u_{p} / U_{1}$, and the dashed line is the normalized viscous component $\bar{u}_{v}=u_{v} / U_{1}$. It is clear that the viscous component is in phase with the velocity of the cylinder when evaluated at the cylinder surface $(\bar{r}=1)$. On the other hand, the potential component is nearly $\pi$ out of phase with the cylinder velocity.

The spatial variation of the phase differences are shown for $\beta=1.26$ in Fig. 5(b). The phase difference $\phi_{y}$ is shown as the solid line. The phase $\phi_{y}$ is zero at $\bar{r}=1$ and then
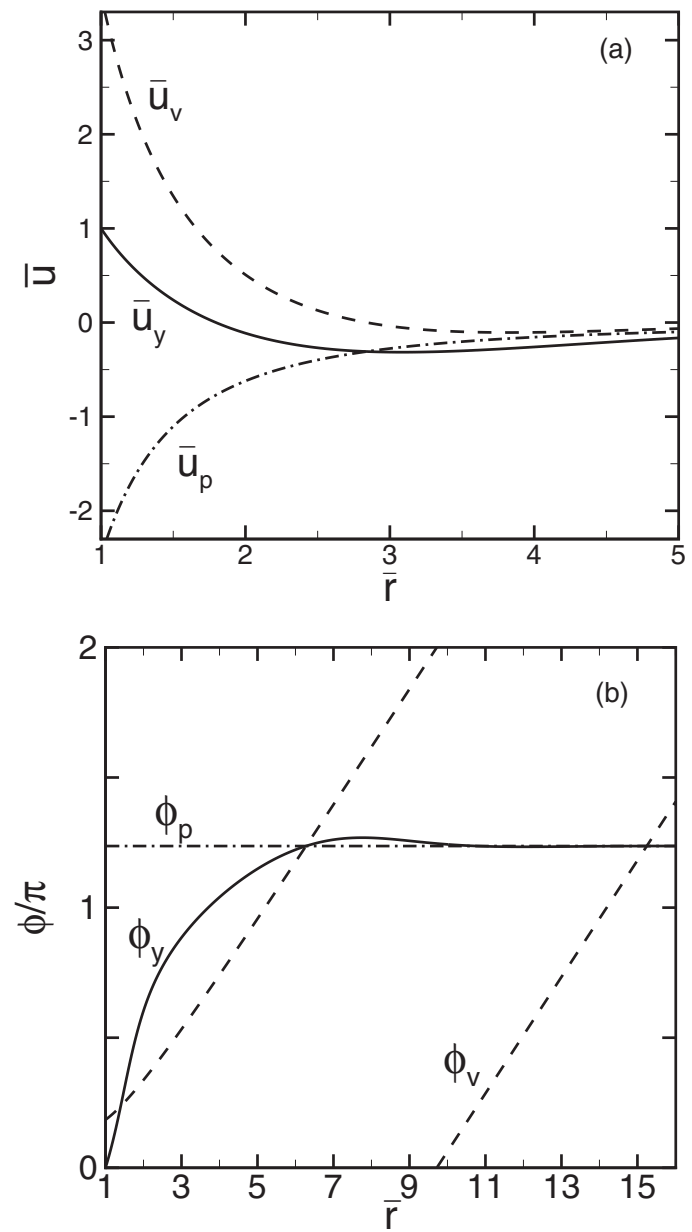

FIG. 5. The variation of the fluid velocity and its phase, relative to the velocity of the oscillating cylinder, with normalized radial distance $\bar{r}=r / a$ at time $t=0$, where $\beta=1.26$. (a) The variation of the fluid velocity where the solid line is $\bar{u}_{y}=u_{y} / U_{1}$, the dashed line is the viscous component $\bar{u}_{v}=u_{v} / U_{1}$, and the dash-dotted line is the potential component $\bar{u}_{p}=u_{p} / U_{1}$. (b) The phase $\phi$ of the fluid velocity relative to the cylinder velocity. The solid line is for the fluid velocity $\phi_{y}$, the dashed line is for the viscous component $\phi_{v}$, and the dash-dotted line is for the potential component $\phi_{p}$.

approaches the value from the potential component $\phi_{p}$ for larger separations. The phase of the potential component is shown as the dash-dotted line. The value of $\phi_{p}$ is independent of $r$ and is weakly dependent on the value of the frequency parameter $\beta$. In the limit of $\beta \rightarrow 0$ the phase $\phi_{p}$ approaches $\pi$. The phase of the viscous component $\phi_{v}$ is shown as the dashed line. The slope of the dashed line is nearly constant except for small values of $\bar{r}$ where there is some deviation. The slope of the line describes the motion of the viscous momentum waves that are traveling away from the oscillating cylinder.

\section{THE FORCE ACTING ON AN OSCILLATING CYLINDER DUE TO A VISCOUS FLUID}

Using the solution for the flow field caused by an oscillating cylinder given by Eqs. (12) and (13), we next discuss the forces acting on the cylinder due to the viscous fluid. 


\section{A. The forces acting on an oscillating cylinder in a quiescent background flow field}

The fluid force $F_{f}$ acting on a cylinder of length $L$ that is oscillating at a frequency $\omega$ in a viscous fluid is given by (cf. Ref. [24])

$$
F_{f}=a L \int_{0}^{2 \pi}\left(p_{r r} \cos \theta-p_{r \theta} \sin \theta\right) d \theta,
$$

where $p_{r r}$ and $p_{r \theta}$ are the components of the stress tensor given by

$$
\begin{gathered}
p_{r r}=-p+2 \mu \frac{\partial u_{r}}{\partial r}, \\
p_{r \theta}=\mu\left[r \frac{\partial}{\partial r}\left(\frac{u_{\theta}}{r}\right)+\frac{1}{r} \frac{\partial u_{r}}{\partial \theta}\right] .
\end{gathered}
$$

Using the velocity field given by Eqs. (12) and (13), it can be shown that the pressure in the fluid is given by

$$
p=i \omega U_{1} \rho \frac{A}{r} e^{i \omega t} \cos \theta .
$$

Using these expressions in Eq. (28) and evaluating the result at $r=a$ yields

$$
F_{f}=-i m_{f} \omega U_{1} \Gamma(\beta) e^{i \omega t},
$$

where

$$
m_{f}=\rho \pi a^{2} L
$$

is the mass of fluid displaced by the cylinder and $\Gamma$ is the hydrodynamic function given by

$$
\Gamma(\beta)=1-\frac{4 K_{1}[\sqrt{i \beta}]}{K_{1}[\sqrt{i \beta}]+\sqrt{i \beta} K_{1}^{\prime}[\sqrt{i \beta}]} .
$$

\section{B. The fluid force on oscillating cylinders in an oscillating flow field}

In this case we consider two cylinders oscillating in fluid that are separated by a distance $s$ as shown in Fig. 6. We begin with an inertial reference frame that is stationary where the

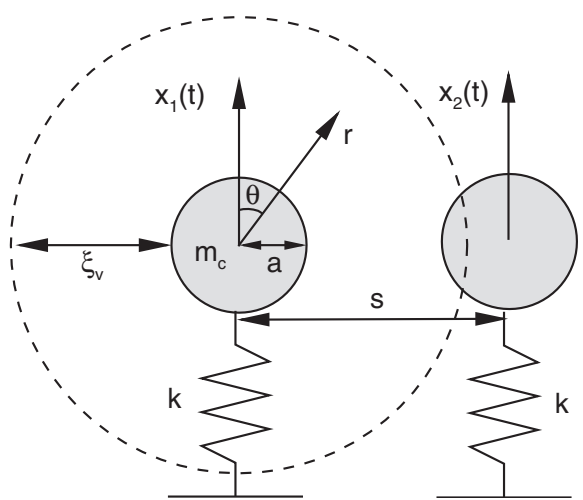

FIG. 6. Two oscillating cylinders of radius $a$ and mass $m_{c}$ immersed in a viscous fluid and separated by a distance $s$. Each cylinder is attached to a spring of stiffness $k$. The time-varying displacements of the two cylinders are $x_{1}(t)$ and $x_{2}(t)$, as indicated. The unsteady viscous boundary layer of the left cylinder is indicated by a dashed line and has a thickness of $\xi_{v}$. motion of the two cylinders is given by

$$
\begin{aligned}
& \vec{u}_{1}=U_{1} e^{i \omega t} \hat{e}_{x}=A_{1} \omega e^{i \omega t} \hat{e}_{x}, \\
& \vec{u}_{2}=U_{2} e^{i \omega t} \hat{e}_{x}=A_{2} \omega e^{i \omega t} \hat{e}_{x} .
\end{aligned}
$$

We seek an expression for the force due to the fluid acting on the cylinder on the right (cylinder 2). We can represent the flow field caused by the oscillations of the left cylinder (cylinder 1) as a background flow field for cylinder 2. This background fluid velocity at the position of cylinder 2 is

$$
\vec{u}_{f}=U_{f} e^{i \omega t} \hat{e}_{x}
$$

where the velocity $U_{f}$ is found by evaluating $u_{y}(r=s)$ using Eq. (18) to yield

$$
U_{f}=-U_{1}\left[\frac{A}{s^{2}}-\frac{B}{a} \sqrt{i \beta} K_{1}^{\prime}\left[\frac{s}{a} \sqrt{i \beta}\right]\right],
$$

which we will rewrite as

$$
U_{f}=-U_{1} \lambda(s),
$$

where the dependence on separation has been collected into $\lambda(s)$.

Therefore, cylinder 2 is an oscillating cylinder in an oscillating background flow field. The flow field is determined by

$$
\begin{gathered}
\rho \frac{\partial \vec{u}}{\partial t}=-\vec{\nabla} p+\mu \nabla^{2} \vec{u}, \\
\vec{\nabla} \cdot \vec{u}=0,
\end{gathered}
$$

with the boundary conditions

$$
\begin{gathered}
u_{r}(r \rightarrow \infty, t)=U_{f} e^{i \omega t} \cos \theta, \\
u_{\theta}(r \rightarrow \infty, t)=-U_{f} e^{i \omega t} \sin \theta, \\
u_{r}(r=a, t)=U_{2} e^{i \omega t} \cos \theta, \\
u_{\theta}(r=a, t)=-U_{2} e^{i \omega t} \sin \theta .
\end{gathered}
$$

We can directly use the solution from Sec. II by moving into a noninertial reference frame attached to the background fluid flow. The fluid velocity in this reference frame is

$$
\overrightarrow{u^{\prime}}=\vec{u}-\vec{u}_{f} .
$$

Inserting Eq. (46) into Eqs. (40) and (41) and rearranging yields the equation for the fluid motion in the noninertial frame,

$$
\begin{gathered}
\rho \frac{\partial \vec{u}^{\prime}}{\partial t}=-\vec{\nabla} p-\rho \frac{d \vec{u}_{f}}{d t}+\mu \nabla^{2} \vec{u}^{\prime}, \\
\vec{\nabla} \cdot \vec{u}^{\prime}=0,
\end{gathered}
$$

where all spatial derivatives of $\vec{u}_{f}$ vanish because it is not a spatially varying flow field. Using the following:

$$
\rho \frac{d \vec{u}_{f}}{d t}=\rho \frac{d u_{f}}{d t} \hat{e}_{x},
$$

where $u_{f}=U_{f} e^{i \omega t}$, we then have

$$
\rho \frac{\partial \vec{u}^{\prime}}{\partial t}=-\vec{\nabla} p^{\prime}+\mu \nabla^{2} \vec{u}^{\prime}
$$


where the modified pressure is

$$
p^{\prime}=p+\rho \frac{d u_{f}}{d t} x .
$$

Therefore, the fluid motion in the noninertial reference frame attached to the oscillating background fluid is given by the solution to Eq. (48) and Eq. (50) with boundary conditions

$$
\begin{gathered}
u_{r}^{\prime}(r \rightarrow \infty, t)=0, \\
u_{\theta}^{\prime}(r \rightarrow \infty, t)=0, \\
u_{r}^{\prime}(r=a, t)=\left(U_{2}-U_{f}\right) e^{i \omega t} \cos \theta, \\
u_{\theta}^{\prime}(r=a, t)=-\left(U_{2}-U_{f}\right) e^{i \omega t} \sin \theta .
\end{gathered}
$$

These equations are the same as Eqs. (6)-(11) used for a single oscillating cylinder in a quiescent fluid with the velocity of the cylinder replaced by $\left(U_{2}-U_{f}\right) e^{i \omega t}$, and the solutions can be carried over from there. The flow field in the noninertial reference frame is given by

$$
\begin{gathered}
u_{r}^{\prime}=\frac{\left(U_{2}-U_{f}\right)}{r}\left(\frac{A}{r}+B K_{1}\left[\frac{r}{a} \sqrt{i \beta}\right]\right) e^{i \omega t} \cos \theta, \\
u_{\theta}^{\prime}=\left(U_{2}-U_{f}\right)\left(\frac{A}{r^{2}}-\frac{B}{a} \sqrt{i \beta} K_{1}^{\prime}\left[\frac{r}{a} \sqrt{i \beta}\right]\right) e^{i \omega t} \sin \theta,
\end{gathered}
$$

where the above expressions are Eqs. (12) and (13) evaluated with a velocity magnitude of $U_{2}-U_{f}$. Furthermore, the pressure in the noninertial frame is given by Eq. (31) using a velocity magnitude of $U_{2}-U_{f}$ to yield

$$
p^{\prime}=i \omega\left(U_{2}-U_{f}\right) \rho \frac{A}{r} e^{i \omega t} \cos \theta .
$$

The fluid force acting on the cylinder contains both viscous and potential contributions and must be calculated in the inertial reference frame. The viscous contribution can be determined using either the flow field in the inertial or noninertial reference frame since it only depends on spatial gradients of the flow field. The pressure in the inertial reference frame is then given by Eq. (51) to yield

$$
p=p^{\prime}-\rho \frac{d u_{f}}{d t} x \text {. }
$$

The force due to the fluid motion in the inertial reference frame $F_{f}$ can then be evaluated using Eq. (28). This force can be decomposed into the following two components:

$$
F_{f}=\tilde{F}_{f}+F_{f}^{\prime},
$$

where $\tilde{F}_{f}$ is Eq. (32) evaluated with a velocity magnitude of $\left(U_{2}-U_{f}\right)$ to yield

$$
\tilde{F}_{f}=-i m_{f} \omega\left(U_{2}-U_{f}\right) \Gamma(\beta) e^{i \omega t}
$$

and $F_{f}^{\prime}$ is the component due to the modified pressure field $p^{\prime}$ that resulted from the noninertial reference frame. Using Eq. (59) in Eq. (29) yields that the normal component of the stress tensor is

$$
p_{r r}=-p^{\prime}+\rho \frac{d u_{f}}{d t} x+2 \mu \frac{\partial u_{r}}{\partial r} .
$$

The additional contribution to the force due to the modified pressure is then

$$
F_{f}^{\prime}=a L \int_{0}^{2 \pi} \rho \frac{d u_{f}}{d t} x \cos \theta d \theta
$$

where

$$
\rho \frac{d u_{f}}{d t} x=-i \rho \omega U_{1} \lambda(s) e^{i \omega t} \frac{a}{\cos \theta} .
$$

Performing the integration and rearranging yields

$$
F_{f}^{\prime}=-2 i m_{f} \omega U_{1} \lambda(s) e^{i \omega t} .
$$

Therefore, the total fluid force acting on cylinder 2 is

$$
F_{f}=-i m_{f} \omega\left(U_{2}-U_{f}\right) \Gamma(\beta) e^{i \omega t}-2 i m_{f} \omega U_{1} \lambda(s) e^{i \omega t} .
$$

Using Eq. (39) for $U_{f}$ and rearranging yields

$$
F_{f}=-i m_{f} \omega\left[U_{2} \Gamma(\beta)+(2+\Gamma(\beta)) U_{1} \lambda(s)\right] e^{i \omega t} .
$$

As expected, in the absence of a background velocity $U_{f}=0$ (or, equivalently, $U_{1}=0$ ) the expression Eq. (67) reduces to Eq. (32).

A similar expression results if one considers the force acting on cylinder 1 in the presence of a background flow field due to the motion of cylinder 2 . We will use the following notation to represent the forces acting on the two cylinders:

$$
\begin{aligned}
& F_{f, 1}=-i m_{f} \omega\left[\dot{x}_{1} \Gamma(\beta)+(2+\Gamma(\beta)) \dot{x}_{2} \lambda(s, \omega)\right], \\
& F_{f, 2}=-i m_{f} \omega\left[\dot{x}_{2} \Gamma(\beta)+(2+\Gamma(\beta)) \dot{x}_{1} \lambda(s, \omega)\right],
\end{aligned}
$$

where $F_{f, 1}$ is the fluid force acting on cylinder 1 and $F_{f, 2}$ is the fluid force acting on cylinder 2 and we have represented the velocity of the two cylinders as

$$
\begin{aligned}
& \dot{x}_{1}=U_{1} e^{i \omega t}, \\
& \dot{x}_{2}=U_{2} e^{i \omega t} .
\end{aligned}
$$

Transforming these expressions into Fourier space yields

$$
\begin{aligned}
& \hat{F}_{f, 1}(\omega)=m_{f} \omega^{2}\left[\hat{x}_{1} \Gamma(\beta)+(2+\Gamma(\beta)) \hat{x}_{2} \lambda(s, \omega)\right], \\
& \hat{F}_{f, 2}(\omega)=m_{f} \omega^{2}\left[\hat{x}_{2} \Gamma(\beta)+(2+\Gamma(\beta)) \hat{x}_{1} \lambda(s, \omega)\right],
\end{aligned}
$$

where we have used the following transform convention:

$$
\begin{gathered}
\hat{f}(\omega)=\int_{-\infty}^{\infty} f(t) e^{-i \omega t} d t, \\
f(t)=\frac{1}{2 \pi} \int_{-\infty}^{\infty} \hat{f}(\omega) e^{i \omega t} d \omega .
\end{gathered}
$$

\section{THE DYNAMICS OF A PAIR OF CYLINDERS IN FLUID}

\section{A. The dynamics when one cylinder is driven externally}

We are ultimately interested in the coupled dynamics of two elastic objects placed in fluid, such as two cantilevers or two nanowires placed in close proximity. A schematic illustrating the general situation is shown in Fig. 6. The fundamental mode of oscillation of the elastic objects is modeled as a lumped mass on a spring. The two cylinders have identical mass $m_{c}$, radius $a$, and spring constant is $k$, although it would be straightforward to vary these. 
The first case we consider in detail is where the cylinder on the left is driven by an external harmonic force $F_{d}=F_{0} e^{i \omega t}$, where $F_{0}$ is the magnitude. Since we are interested in the result over all frequencies we will solve this analytically in the Fourier domain using an impulse in force given by

$$
F_{d}=F_{0} \delta(t) .
$$

Under these conditions the equations of motion become

$$
\begin{gathered}
-m_{c} \omega^{2} \hat{x}_{1}+k \hat{x}_{1}=F_{0}+\hat{F}_{f, 1}(\omega), \\
-m_{c} \omega^{2} \hat{x}_{2}+k \hat{x}_{2}=\hat{F}_{f, 2}(\omega) .
\end{gathered}
$$

This can be rewritten as

$$
\begin{aligned}
& -\omega^{2} \hat{x}_{1}-T_{0} \omega^{2} \Gamma(\beta) \hat{x}_{1}+\omega_{0}^{2} \hat{x}_{1} \\
& =\frac{F_{0}}{m_{c}}+(2+\Gamma(\beta)) T_{0} \omega^{2} \lambda(s, \omega) \hat{x}_{2}(\omega)
\end{aligned}
$$

and

$$
\begin{array}{r}
-\omega^{2} \hat{x}_{2}-T_{0} \omega^{2} \Gamma(\beta) \hat{x}_{2}+\omega_{0}^{2} \hat{x}_{2} \\
=(2+\Gamma(\beta)) T_{0} \omega^{2} \lambda(s, \omega) \hat{x}_{1},
\end{array}
$$

where the mass loading parameter is

$$
T_{0}=\frac{m_{f}}{m_{c}} .
$$

This can be solved to yield

$$
\begin{gathered}
\hat{\bar{x}}_{1}(\tilde{\omega})=\frac{1+\left(2+\Gamma\left(\beta_{0} \tilde{\omega}\right)\right) T_{0} \tilde{\omega}^{2} \lambda\left(\bar{s}, \tilde{\omega}, \beta_{0}\right) \hat{\bar{x}}_{2}}{\left(1-\tilde{\omega}^{2}\right)-T_{0} \tilde{\omega}^{2} \Gamma\left(\beta_{0} \tilde{\omega}\right)}, \\
\hat{\bar{x}}_{2}(\tilde{\omega})=\frac{\left(2+\Gamma\left(\beta_{0} \tilde{\omega}\right)\right) T_{0} \tilde{\omega}^{2} \lambda\left(\bar{s}, \tilde{\omega}, \beta_{0}\right) \hat{\bar{x}}_{1}}{\left(1-\tilde{\omega}^{2}\right)-T_{0} \tilde{\omega}^{2} \Gamma\left(\beta_{0} \tilde{\omega}\right)},
\end{gathered}
$$

where $\omega_{0}^{2}=k / m_{c}, \beta_{0}=\omega_{0} a^{2} / \nu$, and $\tilde{\omega}=\omega / \omega_{0}$. The cylinder displacements $x_{1}$ and $x_{2}$ have been nondimensionalized as

$$
\begin{aligned}
& \bar{x}_{1}=x_{1} / x_{f}, \\
& \bar{x}_{2}=x_{2} / x_{f},
\end{aligned}
$$

where $x_{f}=F_{0} / k$ and the term $\lambda(s, \omega)$ has been rewritten as $\lambda\left(\bar{s}, \tilde{\omega}, \beta_{0}\right)$, where

$$
\lambda\left(\bar{s}, \tilde{\omega}, \beta_{0}\right)=\frac{\bar{A}}{\bar{s}^{2}}-\bar{B} \sqrt{i \beta_{0} \tilde{\omega}} K_{1}^{\prime}\left[\bar{s} \sqrt{i \beta_{0} \tilde{\omega}}\right],
$$

using the following definitions:

$$
\begin{gathered}
\bar{s}=s / a, \\
\bar{A}=A / a^{2}, \\
\bar{B}=B / a .
\end{gathered}
$$

Solving for $\hat{\bar{x}}_{1}$ and $\hat{\bar{x}}_{2}$ directly yields

$$
\hat{\bar{x}}_{1}(\tilde{\omega})=\frac{1-\tilde{\omega}^{2}\left[1+T_{0} \Gamma\left(\beta_{0} \tilde{\omega}\right)\right]}{\left(1-\tilde{\omega}^{2}\left[1+T_{0} \Gamma\left(\beta_{0} \tilde{\omega}\right)\right]\right)^{2}-\left(\left(2+\Gamma\left(\beta_{0} \tilde{\omega}\right)\right) T_{0} \tilde{\omega}^{2} \lambda\right)^{2}}
$$

and

$$
\hat{\bar{x}}_{2}(\tilde{\omega})=\frac{\left(2+\Gamma\left(\beta_{0} \tilde{\omega}\right)\right) T_{0} \tilde{\omega}^{2} \lambda}{\left(1-\tilde{\omega}^{2}\left[1+T_{0} \Gamma\left(\beta_{0} \tilde{\omega}\right)\right]\right)^{2}-\left(\left(2+\Gamma\left(\beta_{0} \tilde{\omega}\right)\right) T_{0} \tilde{\omega}^{2} \lambda\right)^{2}} .
$$

The expression for the dynamics of a single cylinder in the absence of a neighboring cylinder can by found directly by setting $\hat{\bar{x}}_{2}=0$ in Eq. (82) to yield

$$
\hat{\bar{x}}_{1}(\tilde{\omega})=\frac{1}{1-\tilde{\omega}^{2}\left(1+T_{0} \Gamma\left(\beta_{0} \tilde{\omega}\right)\right)} .
$$

\section{B. The dynamics of a pair of cylinders in fluid driven by Brownian motion}

The stochastic dynamics of a cylinder pair in fluid can be found using the fluctuation-dissipation theorem and linear response theory $[19,20]$. The essence of the approach is to perturb the system and compute its return to equilibrium. The most transparent way to use this approach is to apply a constant force displacing one of the cylinders at some time in the distant past which represents some prescribed perturbation from equilibrium. The force is then removed at time $t=0$ and the cylinder returns to equilibrium. The motion of the adjacent cylinder is due to the fluid motion resulting in their correlated motion. Specifically, the deterministic ring down of the cylinder is directly related to the equilibrium autocorrelations in cylinder displacement by

$$
\left\langle x_{1}(0) x_{1}(t)\right\rangle=k_{B} T \frac{X_{1}(t)}{F_{1}},
$$

where $F_{1}$ is the magnitude of the step force applied, $k_{B}$ is Boltzmann's constant, and $T$ is the temperature. In our notation $X_{1}$ represents the deterministic ring down and $x_{1}$ is the stochastic displacement. Similarly, the cross-correlation in the cylinder displacement is given by

$$
\left\langle x_{1}(0) x_{2}(t)\right\rangle=k_{B} T \frac{X_{2}(t)}{F_{1}} .
$$

The cosine Fourier transform of the autocorrelation and cross-correlation yield the noise spectra $G_{11}(\omega)$ and $G_{12}(\omega)$, respectively.

However, it is more convenient in developing analytical expressions for the stochastic dynamics to recall that the noise spectra are related to the complex susceptibilities by

$$
\begin{aligned}
& G_{11}(\omega)=\frac{4 k_{B} T}{\omega} \chi_{11}^{\prime \prime}(\omega), \\
& G_{12}(\omega)=\frac{4 k_{B} T}{\omega} \chi_{12}^{\prime \prime}(\omega) .
\end{aligned}
$$

In these expressions $\chi^{\prime \prime}$ is the imaginary part of the susceptibility, where $\chi$ is the Fourier transform of the deterministic response of the cylinder to the application of an impulse in force. In our notation, $\chi_{11}=-\hat{x}_{1}^{\prime \prime}(\omega)$ and $\chi_{12}=-\hat{x}_{2}^{\prime \prime}(\omega)$ where the double prime indicates the imaginary part. Using the expressions for $\hat{x}_{1}(\omega)$ and $\hat{x}_{2}(\omega)$ from Eqs. (90) and (91) in Eqs. (95) and (96) we have our desired result,

$$
\begin{aligned}
G_{11}(\omega) & =-\frac{4 k_{B} T}{\omega} \hat{x}_{1}^{\prime \prime}(\omega), \\
G_{12}(\omega) & =-\frac{4 k_{B} T}{\omega} \hat{x}_{2}^{\prime \prime}(\omega) .
\end{aligned}
$$


This can be written nondimensionally as

$$
\begin{aligned}
& \bar{G}_{11}(\tilde{\omega})=-\frac{1}{\tilde{\omega}} \hat{\bar{x}}_{1}^{\prime \prime}(\tilde{\omega}), \\
& \bar{G}_{12}(\tilde{\omega})=-\frac{1}{\tilde{\omega}} \hat{\bar{x}}_{2}^{\prime \prime}(\tilde{\omega}),
\end{aligned}
$$

where

$$
\bar{G}=\alpha^{-1} G .
$$

and $\alpha$ is the constant $\alpha=4 k_{B} T / k \omega_{0}$. The noise spectrum for a single cylinder in the absence of a neighboring cylinder is given directly by inserting Eq. (92) in Eq. (99).

\section{DISCUSSION}

In this section we use our expressions to explore the fluid-coupled dynamics of a cylinder pair over a wide range of system parameters. Our discussion is for a cylinder pair in the general configuration shown in Fig. 6. At this point we would like to draw attention to the approximations in our analysis. First is that we have assumed that the Reynolds number $\operatorname{Re}=0$, which is not strictly true. However, for many micro- or nanoscale applications of interest the magnitude of the Reynolds number is negligible (cf. Ref. [26]). As discussed in Sec. II, the amplitude of oscillation for Brownian-driven dynamics is usually very small (nanometers or smaller), which leads to negligible values of the Reynolds number for typical frequencies of oscillation in common fluids of interest. For the case of externally driven dynamics one can tailor the amplitude and frequency of the dynamics to always ensure a small value of the Reynolds number. As an example, an elastic object with a characteristic length of $1 \mu \mathrm{m}$ oscillating in water at $1 \mathrm{MHz}$ with an amplitude of $1 \mathrm{~nm}$ would have a Reynolds number of $\operatorname{Re} \approx 10^{-3}$. However, one could imagine situations where this is not true, such as a very soft nanoscale cantilever undergoing large deflection dynamics or perhaps a cantilever that is driven externally to have a large amplitude. It would be interesting to understand how our results would change in this limit. As the Reynolds number becomes important there would be additional sources of fluid dissipation and flow structures such as eddies and vortices which would affect the results. Effects such as these are not included in our present analysis.

An additional assumption underlying our expressions for the fluid force acting on an oscillating cylinder is that we have treated the cylinder as stationary and located at its mean position of zero displacement when solving the fluid equations. However, it is possible to show that this approximation is to the same order as the linearization of the fluid equations (cf. Ref. [24]).

The most significant approximation in our analysis is in the way we compute the fluid force on each cylinder due to the flow field of its neighbor. We treat the flow field from the neighboring cylinder as a spatially uniform and time-varying background flow field. In essence our approach is a method of reflection approach [27] where we have included a single reflection. Specifically, the background flow field is chosen to be the flow field a distance $s$ away from the center of mass of an oscillating neighbor. We have accounted for the back action only in an approximate way. The flow field around a cylinder does include a contribution due to the oscillating neighbor.
However, we have not accounted for the spatial variation in the flow field from a neighbor that is acting on the cylinder. For example, the flow field acting on the edge of the cylinder closer to its neighbor will be different from the edge that is further away. In addition, we have not accounted for the effect of the finite size of the neighboring cylinder on the streamlines of the flow field. We would expect our approximations to be good when the cylinder separation is large compared to the length scale describing the potential component of the flow field $\xi_{p}$.

\section{A. Comparison with numerical simulation}

In this section we compare our theoretical predictions with finite-element simulations in order to shed insight upon the significance of the assumptions made in our theoretical approach. Our time-dependent two-dimensional numerical simulations use a finite-element approach to solve the complete fluid-solid interaction problem for the precise geometry of interest. All of our finite-element numerical simulations have been done using the software available from Ref. [28]. We emphasize that the numerical simulations solve the complete fluid-solid interaction problem that includes the Navier-Stokes equations coupled with the equations of elasticity for the cylinders. We have performed numerous tests to ensure the accuracy and validity of our numerical simulations including temporal and spatial convergence tests.

In particular, we consider the case of two cylinders separated by a distance of $\bar{s}=4$ over a range of frequency parameters $\beta_{0}$. For convenience we have kept the mass loading parameter constant at $T_{0}=1$. The specific geometry explored is shown in Fig. 6. In the numerical simulations the cylinders are immersed in a viscous fluid which is surrounded by walls on which we have applied the no-slip boundary condition. The walls are located very far away from the cylinders to ensure they do not effect the cylinder dynamics through their interactions with the fluid. In all of our simulations the bounding walls are at least a distance of $20 \delta_{0}$ away from the cylinders. The springs that are attached to the cylinders are represented as a Hookean boundary condition on the bottom of each cylinder.

In the numerical simulations used in our comparison we explore the stochastic motion of the two cylinders when driven by Brownian motion. In this case we use the fluctuation dissipation theorem to directly compute the stochastic dynamics using only deterministic calculations. Specifically, a force of magnitude $F_{1}$ is applied to one of the cylinders in the distant past which is removed at time $t=0$. It is convenient to call the cylinder where the force is removed cylinder 1 . The deterministic motion of the cylinders as they return to equilibrium is precisely $X_{1}(t)$ and $X_{2}(t)$. Therefore the auto and crosscorrelation of the equilibrium fluctuations in cylinder displacement is given directly by Eqs. (93) and (94), respectively. The noise spectra $G_{11}(\omega)$ and $G_{12}(\omega)$ can be immediately obtained by taking the Fourier transform of the correlation functions as discussed in Ref. [19]. The theoretical predictions for the auto and cross-correlation in the equilibrium cylinder displacement is obtained by taking the real part of the inverse Fourier transform of the noise spectra given by Eqs. (99) and (100), respectively. In our numerical simulations we typically choose the magnitude of $F_{1}$ such that $X_{1}(0) / a=0.1$. 

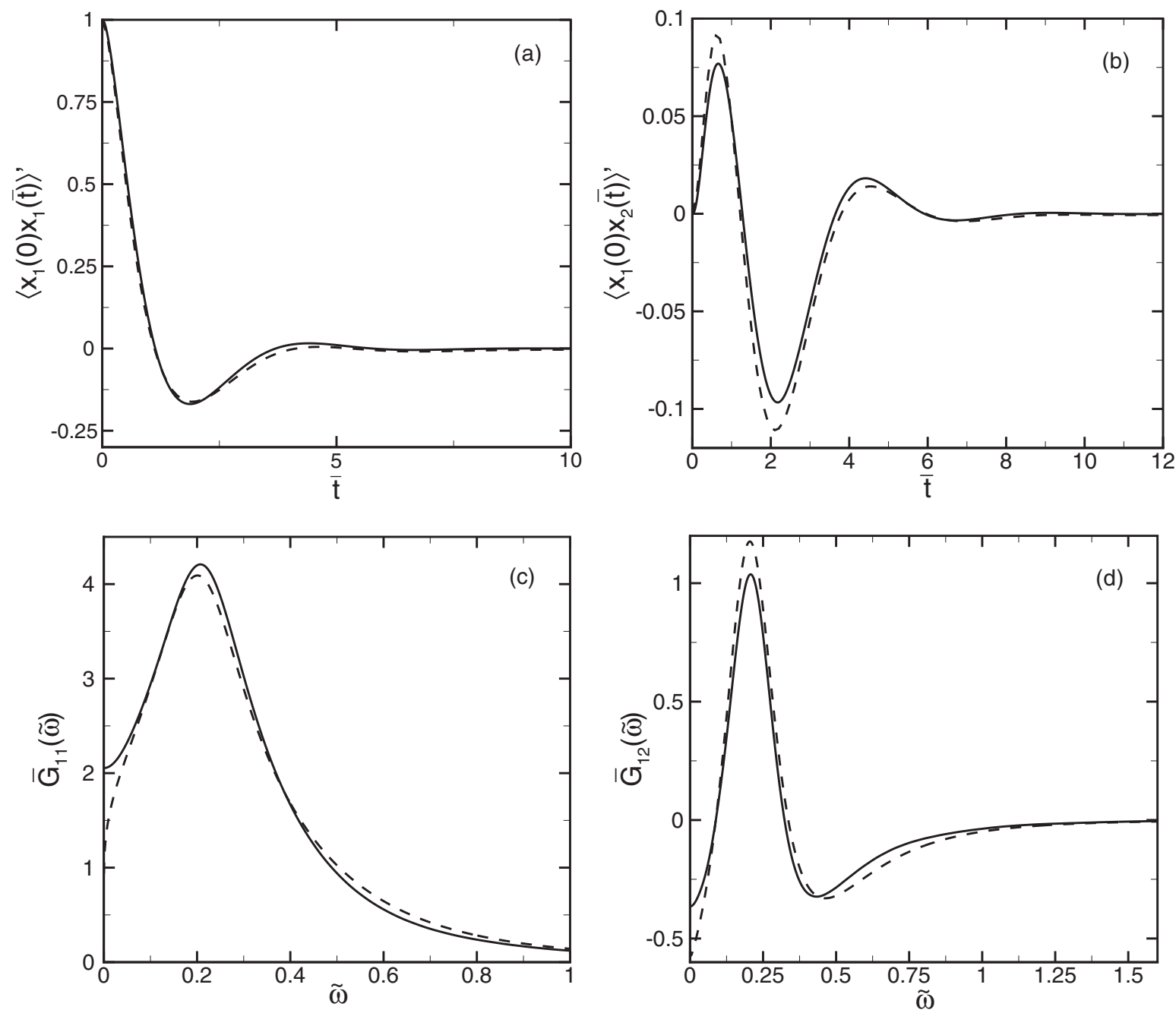

FIG. 7. A comparison between numerical simulations and theoretical predictions for $\beta_{0}=1, T_{0}=1$, and $\bar{s}=4$. The solid lines are from finite-element numerical simulations and the dashed lines are theoretical predictions. (a) The variation of the autocorrelation with time. The theoretical prediction is given by the real part of the inverse Fourier transform of Eq. (99). (b) The variation of the cross-correlation with time. The theoretical prediction is given by the real part of the inverse Fourier transform of Eq. (100). The auto- and cross-correlations have been normalized by a constant value of $k_{b} T / k$ and is indicated by the prime. The normalized time is given by $\bar{t}=t / t_{0}$, where $t_{0}=2 \pi / \omega_{0}$ is the time for one oscillation of in vacuum. (c) The variation of the noise spectrum $\bar{G}_{11}(\tilde{\omega})$ with frequency. The theoretical prediction is given by Eq. (99). (d) The variation of the noise spectrum $\bar{G}_{12}(\tilde{\omega})$ with frequency. The theoretical prediction is given by Eq. (100).

Figure 7 illustrates the comparison between numerical simulation and theory for a frequency parameter of $\beta_{0}=$ 1. These parameters $\left(\beta_{0}=1, T_{0}=1, \bar{s}=4\right)$ are relevant to recent experimental explorations using micron-scale elastic objects in viscous fluid [11,22]. It is useful to recall that the magnitude of the frequency parameter determines the relative size of the unsteady viscous boundary layer as $\delta_{0} / a=\beta_{0}^{-1 / 2}$. Figure 7(a) shows the time variation of the autocorrelation. The normalized time $\bar{t}=t / t_{0}$, where $t_{0}=$ $2 \pi / \omega_{0}$, and the autocorrelation has been scaled by $k_{b} T / k$ and is indicated by the prime notation. Figure 7(b) shows the comparison for the cross-correlation and Figs. 7(c) and 7(d) show the noise spectra. The solid lines are from numerical simulation and the dashed lines are found using our analytical expressions. Overall the agreement between the theoretical predictions and the numerical simulations is very good. This suggests that the approximations used in our analysis do not significantly affect the results for the parameter range of interest.

For these parameters Fig. 7 indicates that the dynamics of the cylinders is slightly underdamped, which results in both positive and negative values of the auto- and cross-correlation functions. This yields a noise spectrum $\bar{G}_{11}$ with a well-defined peak and a noise spectrum $\bar{G}_{12}$ with positive and negative values. The physical implications of the shape of the noise spectrum $\bar{G}_{12}$ will be explored further in the upcoming discussion. These results are in qualitative agreement with the correlated dynamics of two closely spaced atomic force microscopy cantilevers that are placed end to end in a viscous fluid $[11,20,22]$.

Next, we explore the comparison between theory and numerics over a range of frequency parameters that covers two orders of magnitude $1 \leqslant \beta_{0} \leqslant 100$. In these results the cylinder separation and mass loading remain constant at 

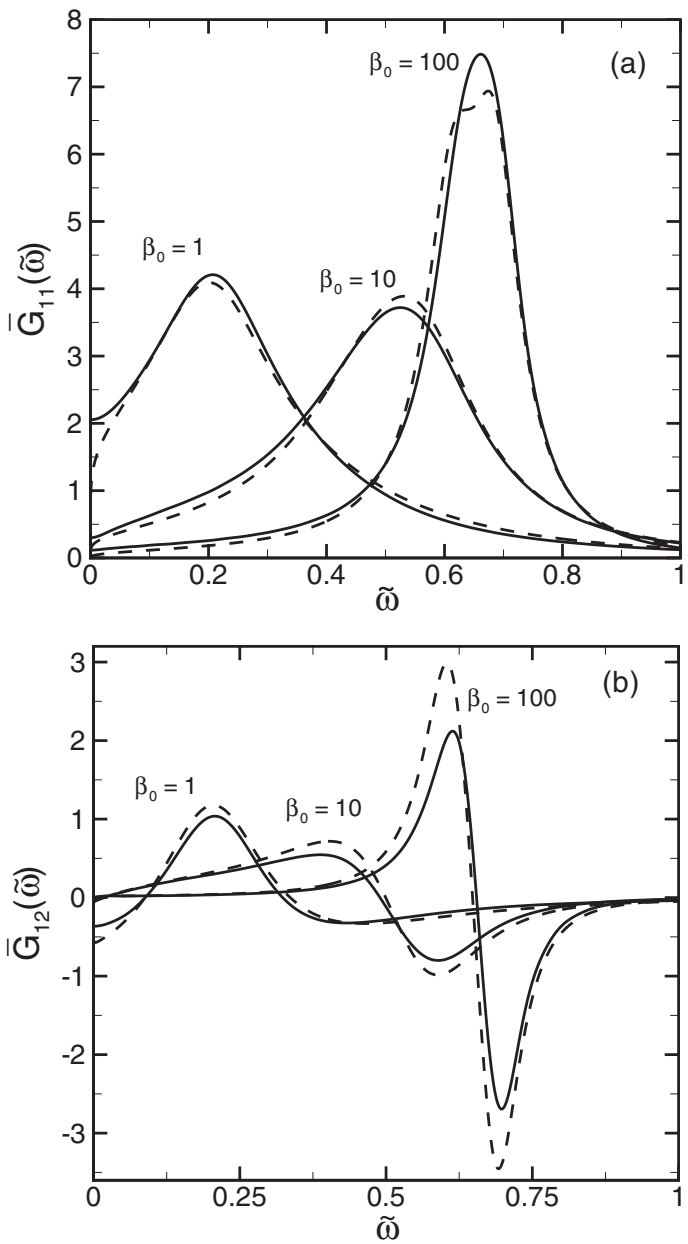

FIG. 8. A comparison between numerical simulations and theoretical predictions of the noise spectra over a range of frequency parameters $\beta_{0}$ that covers two orders of magnitude where $T_{0}=1$ and $\bar{s}=4$. The solid lines are from finite-element numerical simulations and the dashed lines are theoretical predictions for $\bar{G}_{11}(\tilde{\omega})$ and $\bar{G}_{12}(\tilde{\omega})$ using Eqs. (99) and (100), respectively. (a) The variation of $\bar{G}_{11}(\tilde{\omega})$; (b) the variation of $\bar{G}_{12}(\tilde{\omega})$.

$\bar{s}=4$ and $T_{0}=1$. We emphasize that varying the frequency parameter strongly affects the nature of the fluid interactions between the cylinders. As $\beta_{0}$ increases the viscous boundary layer becomes smaller. As a result, this becomes difficult to simulate numerically in the large $\beta_{0}$ limit. This is due, in part, to the spatial resolution requirement to accurately represent both the large gradients in the small viscous boundary layer and the long-range fluid dynamics typical of low-Reynolds-number flows. Figure 8 shows the comparison between theory and numerics for the noise spectra $\bar{G}_{11}$ and $\bar{G}_{12}$. The agreement between theory and numerics is quite good. In the following sections we use the theoretical expressions to explore the fluid-coupled dynamics of a pair of cylinders over a broad range of conditions.

\section{B. The fluid-coupled motion of two cylinders when one cylinder is driven externally}

We now discuss the variation in the cylinder dynamics when one cylinder is driven by a harmonic external actuation force and the adjacent cylinder is passive in that its motion is driven by the dynamics of the surrounding fluid. We emphasize that the dynamics of the cylinder pair is determined by their mutual fluid-solid interactions.

We first consider the case where the two cylinders are held at a constant separation of $\bar{s}=6$. Using our notation this corresponds to a dimensional distance of $4 a$ from the surface of one cylinder to the surface of the adjacent cylinder along the line that connects their two centers. We next explore the effect of the mass loading parameter $T_{0}$ on the dynamics while holding the frequency parameter $\beta_{0}$ and the cylinder separation $\bar{s}$ constant. The results are shown in Fig. 9 where panel (a) illustrates the response of the driven cylinder $\left|\hat{\bar{x}}_{1}(\tilde{\omega})\right|$ and panel (b) illustrates the response of the passive adjacent cylinder $\left|\hat{\bar{x}}_{2}(\tilde{\omega})\right|$ where we have used Eqs. (90) and (91). It is
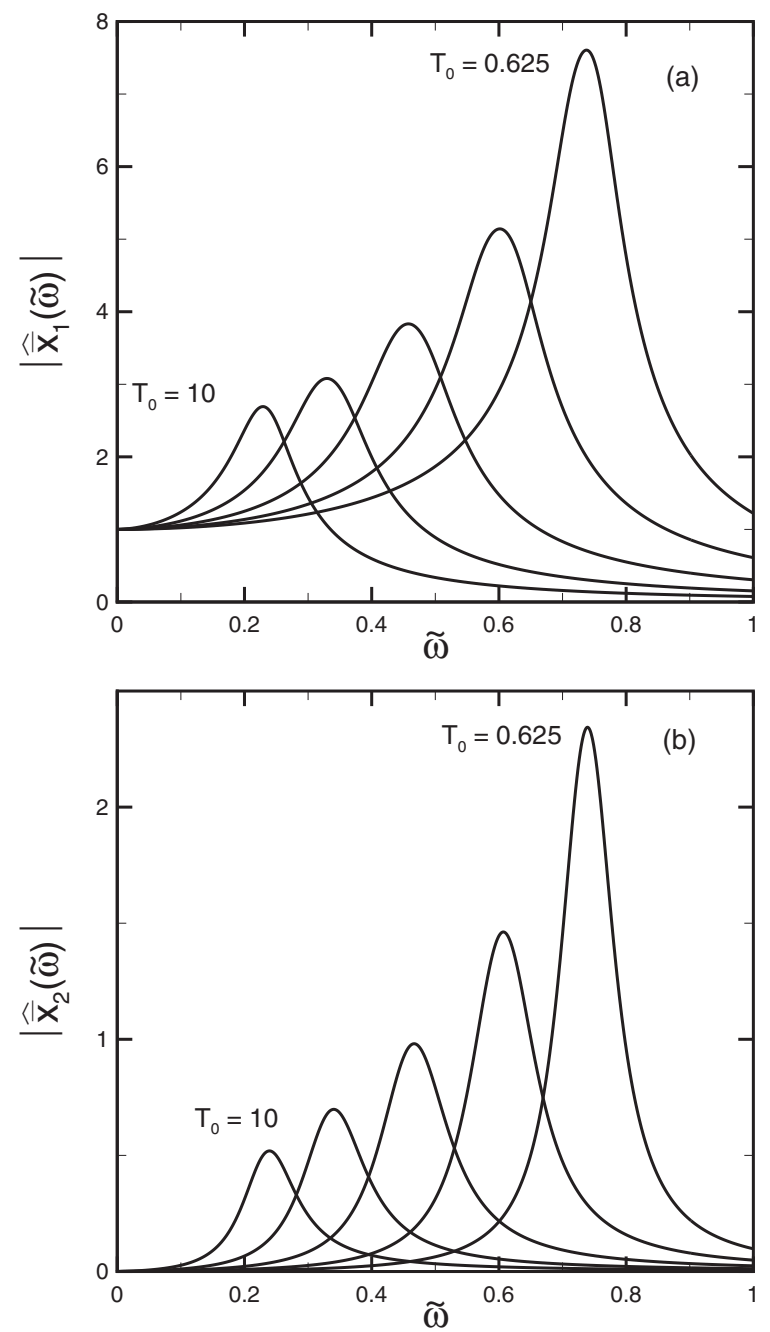

FIG. 9. The variation of the amplitude of oscillation with reduced frequency of a cylinder pair where one cylinder is driven externally. For these results the frequency parameter is $\beta_{0}=100$, the cylinder separation is $\bar{s}=6$, and the different solid curves are for the mass loading parameters $T_{0}=0.625,1.25,2.5,5,10$. The smallest and largest values of $T_{0}$ are labeled with the remaining values in sequence. (a) The variation of the amplitude of the driven cylinder $\left|\hat{\bar{x}}_{1}(\tilde{\omega})\right|$ given by Eq. (90). (b) The variation of the amplitude of oscillation of the passive cylinder $\left|\hat{\bar{x}}_{2}(\tilde{\omega})\right|$ given by Eq. (91). 
clear that as the mass loading parameter increases, the general signature of the response does not change significantly. As the mass of the fluid becomes larger relative to the mass of the elastic object the amplitude of the response decreases and the resonant frequency shifts towards lower frequencies. For typical micron and nanoscale geometries that are of interest for the development of new technologies that could exhibit or exploit fluid coupled dynamics, $0.1 \lesssim T_{0} \lesssim 10$. For example, for a silicon cylinder oscillating in water the value of the mass loading parameter is $T_{0}=0.43$, for a nanoscale cantilever made of silicon and immersed in water $T_{0} \approx 1$, and for a typical atomic force microscope cantilever in water $T_{0} \approx 5$.

Figure 10 illustrates the variation of the power spectral densities $\left|\hat{\bar{x}}_{1}(\tilde{\omega})\right|$ and $\left|\hat{\bar{x}}_{2}(\tilde{\omega})\right|$ with the frequency parameter

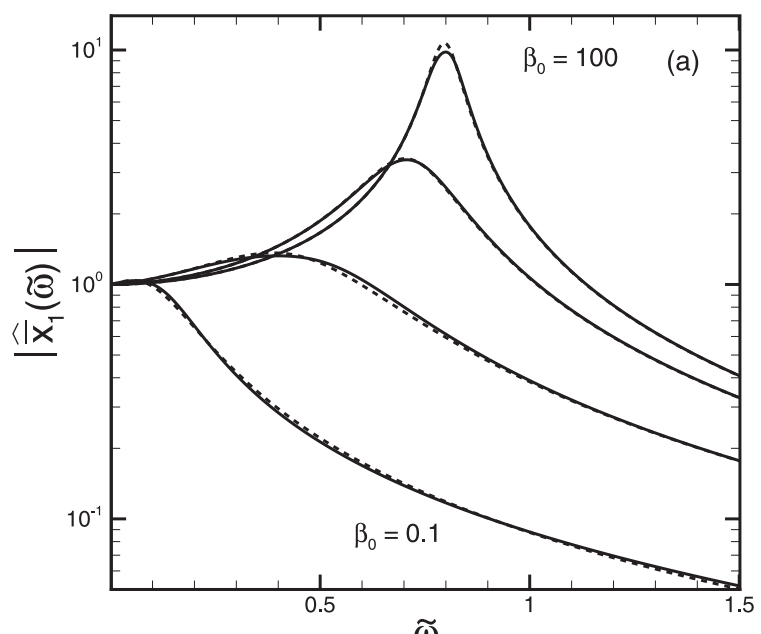

$\widetilde{\omega}$

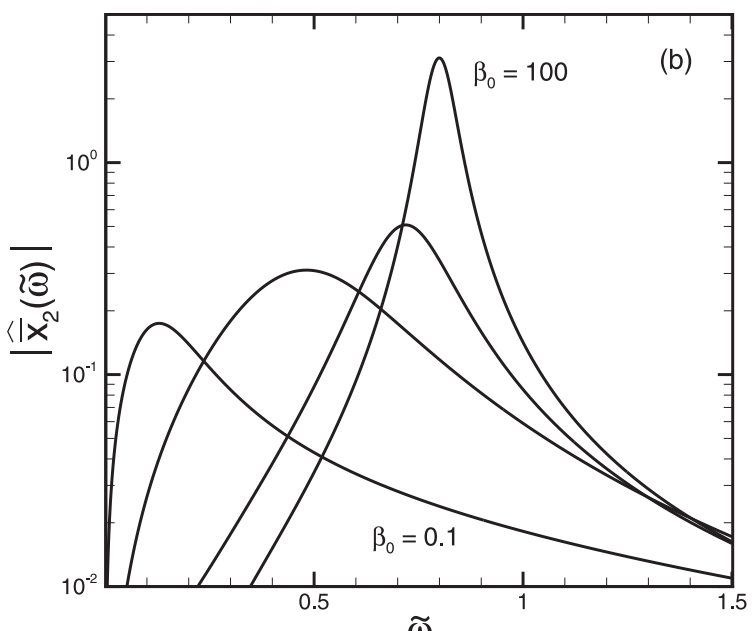

$\tilde{\omega}$

FIG. 10. The variation of the amplitude of oscillation with reduced frequency of a cylinder pair where one cylinder is driven externally in the presence of a passive adjacent cylinder. For these results the mass loading parameter is $T_{0}=0.43$, the cylinder separation is $\bar{s}=6$, and the different solid curves are for $\beta_{0}=0.1,1,10,100$. The smallest and largest values of $\beta_{0}$ are labeled with the remaining values in sequence. (a) The variation of the amplitude of the driven cylinder $\left|\hat{\bar{x}}_{1}(\tilde{\omega})\right|$. The solid lines are found using Eq. (90). The dashed lines are for the case of a single cylinder in an unbounded fluid using Eq. (92). (b) The variation of the amplitude of oscillation of the passive cylinder $\left|\hat{\bar{x}}_{2}(\tilde{\omega})\right|$ given by Eq. (91). $\beta_{0}$. The dynamics are shown over a range of three orders of magnitude in the frequency parameter where $0.1 \leqslant \beta_{0} \leqslant 100$. For convenience we have also used a constant value of the mass loading parameter of $T_{0}=0.43$ corresponding to a silicon cylinder oscillating in water. The solid lines are the predictions of the dynamics using Eqs. (90) and (91). The dashed lines of Fig. 10(a) illustrate the predictions for a single cylinder in an unbounded fluid without a neighboring cylinder given by Eq. (92). The frequency parameter is a nondimensionalization of the resonant frequency and represents the ratio of local inertial forces to viscous forces. For small values of the frequency parameter viscous forces are strong and for large values of the frequency parameter the dynamics are dominated by potential interactions. Over the range of the frequency parameter shown in Fig. 10 the response can be described by a single peak. Furthermore, the dashed lines and solid lines of Fig. 10(a) show very little deviation, indicating that the presence of the neighboring cylinder has very little influence on the dynamics of the driven cylinder. As the frequency parameter is reduced, and therefore the viscous interactions increase, the magnitude of the peak diminishes and shifts to lower frequencies as expected.

The cylinder dynamics can be elucidated further by quantifying the variation in the dynamics with increasing cylinder separation. Figure 11 illustrates the variation of the power spectral densities with cylinder separation where the frequency parameter and mass loading parameter are held constant. The frequency parameter is held at $\beta_{0}=10^{3}$, the mass loading parameter is $T_{0}=0.43$, and the cylinder separation is over the range $4 \leqslant \bar{s} \leqslant 10$. Figure 11(a) shows the power spectral density of the cylinder that is driven. The dashed line is the theoretical prediction for the dynamics of the driven cylinder in an unbounded fluid without a neighboring cylinder and is found by evaluating Eq. (92). The solid lines are the results for the driven cylinder when a second cylinder is located at a distance of $\bar{s}$. As the cylinder separation $\bar{s}$ is reduced the peak in $\left|\hat{\bar{x}}_{1}(\tilde{\omega})\right|$ splits into two, suggesting the presence of two modes in the dynamics. A similar trend is seen in Fig. 11(b) for the variation of $\left|\hat{\bar{x}}_{2}(\tilde{\omega})\right|$ with the cylinder separation.

In order to describe the general features of the dynamics, and the mode splitting that is exhibited, we explore in more detail two important limiting regimes. For $\beta_{0} \rightarrow \infty$ the unsteady viscous boundary layer reduces to zero thickness while satisfying the no-slip boundary condition to yield purely potential or ideal fluid dynamics. In this case, the two cylinders only interact through the potential contributions of their flow fields. However, in the limit of $\beta_{0} \rightarrow 0$ the unsteady viscous boundary layer grows without bound. In this limit the two cylinders reside entirely within the viscous boundary layer of its neighbor, resulting in strong viscous interactions. The dynamics of the cylinder pair can be understood in terms of the interactions of the viscous and potential components of the flow field and the cylinders.

\section{The viscous and potential contributions to the fluid-coupled dynamics}

We first discuss the results in the potential limit where $\beta_{0} \rightarrow$ $\infty$. In this limit the potential length scale and the viscous length scale become $\xi_{f} / a=10$ and $\xi_{v} / a=1$, respectively. This is 

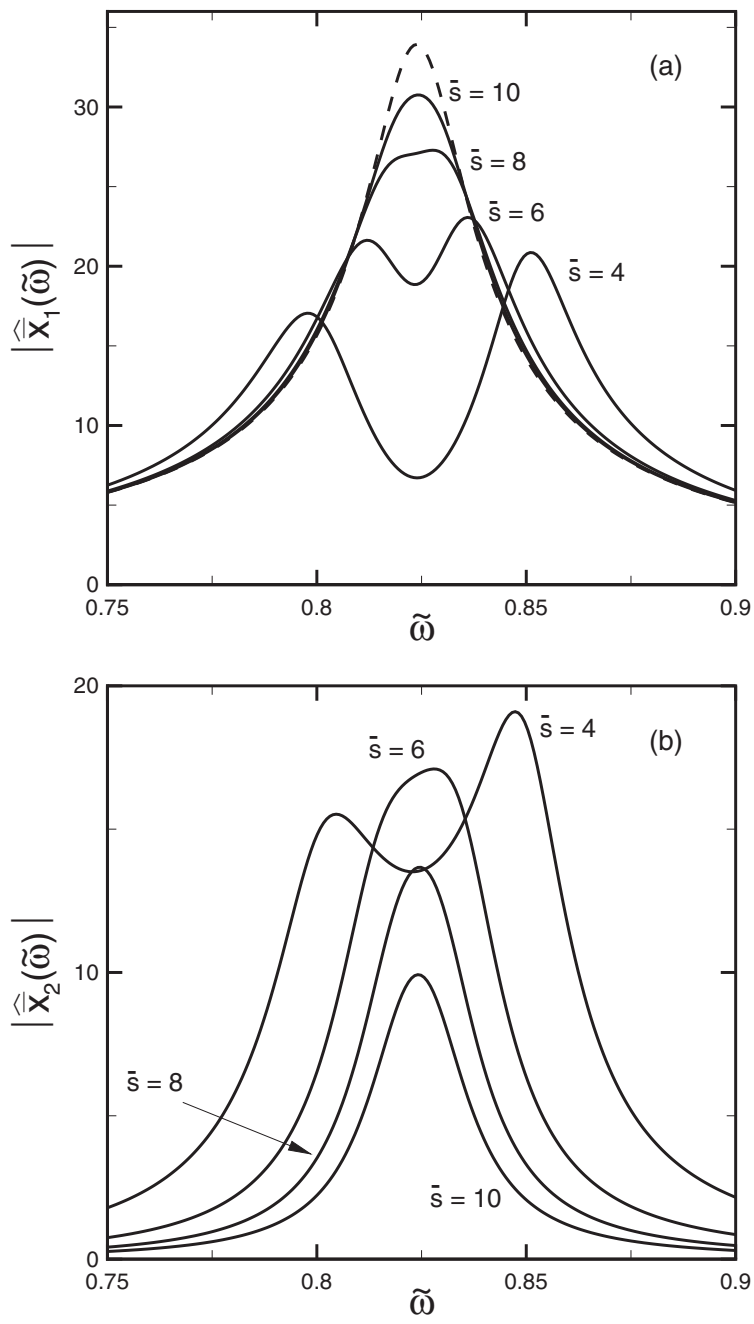

FIG. 11. The amplitude of oscillation of a cylinder pair where one cylinder is driven externally. These results illustrate the variation of the cylinder amplitude with reduced frequency $\tilde{\omega}$ as the separation $\bar{s}$ is increased when $\beta_{0}=10^{3}$ and $T_{0}=0.43$. (a) The amplitude of oscillation for the driven cylinder, $\left|\hat{\bar{x}}_{1}(\omega)\right|$. The solid lines are for the separations $\bar{s}=4,6,8,10$ using Eq. (90) and the dashed line is the amplitude of oscillation in the absence of an adjacent cylinder given by using Eq. (92). (b) The amplitude of oscillation for the passive cylinder $\left|\hat{\bar{x}}_{2}(\omega)\right|$ found using Eq. (91).

evident from Fig. 4(a) and the factor of 10 emerges from our choice of determining the length scales using $1 \%$ of $U_{1}$ as the threshold. In addition, in the potential limit the hydrodynamic function becomes $\Gamma=1$, which yields the potential flow field given by

$$
\begin{aligned}
& u_{r}=\frac{U_{1}}{\bar{r}^{2}} e^{i \omega t} \cos \theta, \\
& u_{\theta}=\frac{U_{1}}{\bar{r}^{2}} e^{i \omega t} \sin \theta,
\end{aligned}
$$

which decreases quadratically with the radial distance. Note that $u_{\theta}(r, \theta=\pi / 2, t=0)$ corresponds to the potential velocity given by Eq. (20) where the coefficient $A=a^{2}$ in the potential limit. The fluid interaction between the cylinders is captured by $\lambda$, which becomes $\lambda=\bar{s}^{-2}$ in the potential limit.
An expression for the time variation of the force acting on a single cylinder oscillating at frequency $\omega$ can be found by setting $\Gamma=1$ in Eq. (32) to yield

$$
F_{f}(t)=-i m_{f} \omega U_{1} e^{i \omega t} .
$$

From this expression it can be seen that the fluid contributes only an additional mass term $m_{f}$ which is a constant and given by Eq. (33). Similarly, the fluid force acting on a pair of cylinders is then

$$
\begin{aligned}
& F_{f, 1}(t)=-i m_{f} \omega\left[\dot{x}_{1}+3 \bar{s}^{-2} \dot{x}_{2}\right], \\
& F_{f, 2}(t)=-i m_{f} \omega\left[\dot{x}_{2}+3 \bar{s}^{-2} \dot{x}_{1}\right],
\end{aligned}
$$

which can be written in Fourier space as

$$
\begin{aligned}
& \hat{F}_{f, 1}(\omega)=m_{f} \omega^{2}\left[\hat{x}_{1}+3 \bar{s}^{-2} \hat{x}_{2}\right] \\
& \hat{F}_{f, 2}(\omega)=m_{f} \omega^{2}\left[\hat{x}_{2}+3 \bar{s}^{-2} \hat{x}_{1}\right] .
\end{aligned}
$$

Using the above expressions in the equations of motion [Eqs. (77) and (78)] and solving for $\hat{\bar{x}}_{1}(\tilde{\omega})$ and $\hat{\bar{x}}_{2}(\tilde{\omega})$ directly yields

$$
\begin{aligned}
& \hat{\bar{x}}_{1}(\tilde{\omega})=\frac{1-\tilde{\omega}^{2}\left[1+T_{0}\right]}{\left(1-\tilde{\omega}^{2}\left[1+T_{0}\right]\right)^{2}-\left(\frac{3 T_{0}}{\bar{s}^{2}} \tilde{\omega}^{2}\right)^{2}}, \\
& \hat{\bar{x}}_{2}(\tilde{\omega})=\frac{\frac{3 T_{0}}{\bar{s}^{2}} \tilde{\omega}^{2}}{\left(1-\tilde{\omega}^{2}\left[1+T_{0}\right]\right)^{2}-\left(\frac{3 T_{0}}{\bar{s}^{2}} \tilde{\omega}^{2}\right)^{2}} .
\end{aligned}
$$

The expression for the dynamics of a single cylinder due to potential fluid interactions in the absence of a neighboring cylinder can be found directly by letting $\bar{s} \rightarrow \infty$ in Eq. (109), to yield

$$
\hat{\bar{x}}_{1}(\tilde{\omega})=\frac{1}{1-\tilde{\omega}^{2}\left(1+T_{0}\right)} .
$$

The variation of the amplitude with frequency is shown in Fig. 12 for $\left|\hat{\bar{x}}_{1}(\tilde{\omega})\right|$ and $\left|\hat{\bar{x}}_{2}(\tilde{\omega})\right|$. Our expressions for infinite $\beta_{0}$ are in the absence of damping and therefore the cylinders undergo resonance and have infinite amplitude at the two resonant frequencies.

The equations of motion contain two degrees of freedom and the solution can be written as a sum of normal modes as

$$
\begin{gathered}
\bar{x}_{1}(t)=-\frac{i}{4}\left(\omega_{1} e^{i \omega_{1} t}+\omega_{2} e^{i \omega_{2} t}\right), \\
\bar{x}_{2}(t)=\frac{i}{4}\left(\omega_{1} e^{i \omega_{1} t}-\omega_{2} e^{i \omega_{2} t}\right),
\end{gathered}
$$

where the resonant frequency of the two modes are given by

$$
\begin{aligned}
& \tilde{\omega}_{1}=\left(1+T_{0}-\frac{3 T_{0}}{\bar{s}^{2}}\right)^{-1 / 2}, \\
& \tilde{\omega}_{2}=\left(1+T_{0}+\frac{3 T_{0}}{\bar{s}^{2}}\right)^{-1 / 2} .
\end{aligned}
$$

Equations (112) and (113) represent the time-varying position of the two cylinders where cylinder one experienced an impulse in force at time $t=0$. An expression for the amount of mode splitting in terms of the frequency difference between 

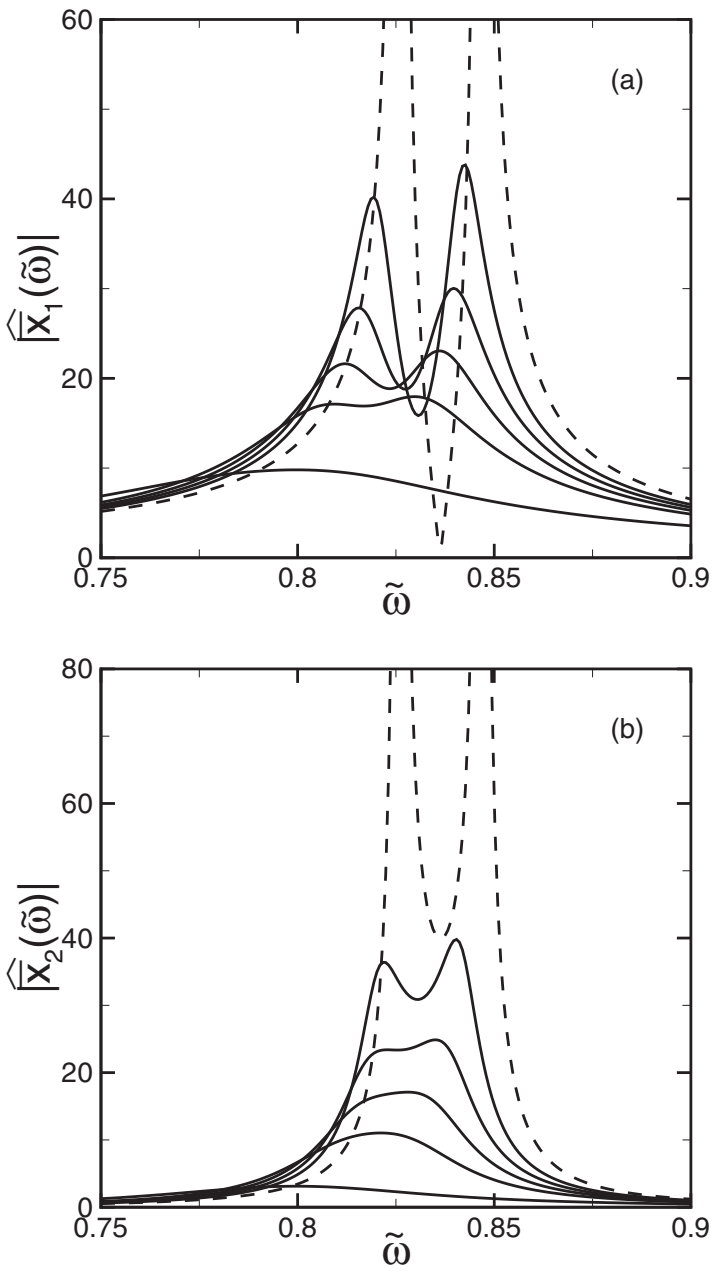

FIG. 12. The amplitude frequency plot for $\left|\hat{\bar{x}}_{1}(\tilde{\omega})\right|$ in panel (a) and $\left|\hat{\bar{x}}_{2}(\tilde{\omega})\right|$ in panel (b) for dynamics dominated by the potential component of the fluid dynamics. The different solid curves are found using Eqs. (90) and (91) for $\beta_{0}=5000,2000,1000,500,100$, where the magnitude of the peak decreases with decreasing values of $\beta_{0}$. The dashed curve is for purely potential fluid dynamics given by $\beta_{0} \rightarrow \infty$ and is found using Eqs. (109) and (110). The remaining parameters used are $\bar{s}=6$ and $T_{0}=0.43$.

the two modes is given by

$$
\Delta \tilde{\omega}=\tilde{\omega}_{1}-\tilde{\omega}_{2}
$$

The two terms on the right-hand side of Eqs. (112) and (113) represent the two normal modes where $\omega_{1}>\omega_{2}$. The highfrequency mode at $\omega_{1}$ is the antisymmetric mode and the lowerfrequency solution at $\omega_{2}$ is the symmetric mode. These two modes can be seen as the resonances in Fig. 12 at $\tilde{\omega}_{1}=0.85$ and $\tilde{\omega}_{2}=0.83$.

Given the response of the cylinders to an impulse in force we can determine the amplitude and phase of the cylinder motion for a harmonic drive at a drive frequency $\omega_{d}$ from

$$
\begin{aligned}
& \bar{x}_{1}(t)=\left|\hat{\bar{x}}_{1}\left(\tilde{\omega}_{d}\right)\right| e^{i\left(\omega_{d} t+\phi_{1}\right)}, \\
& \bar{x}_{2}(t)=\left|\hat{\bar{x}}_{2}\left(\tilde{\omega}_{d}\right)\right| e^{i\left(\omega_{d} t+\phi_{2}\right)},
\end{aligned}
$$

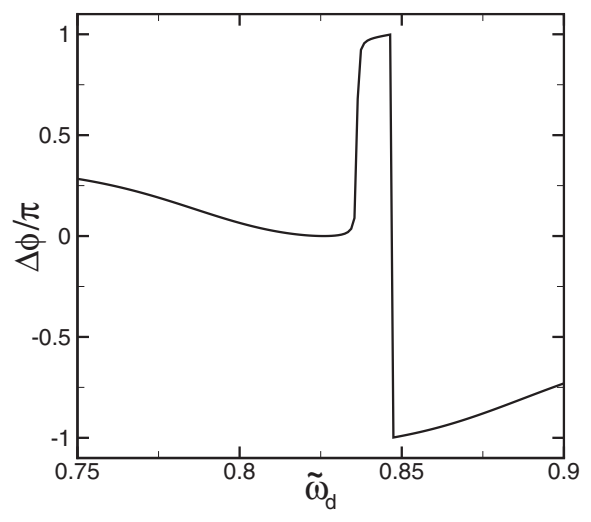

FIG. 13. The variation of the normalized phase difference $\Delta \phi / \pi$ with frequency $\omega_{d} / \omega_{0}$ in the limit of $\beta_{0} \rightarrow \infty$ for purely potential fluid dynamics. The frequency $\omega_{d}$ is the frequency of the harmonic drive force applied to cylinder 1 . The phase difference is $\Delta \phi=\phi_{1}-$ $\phi_{2}$ and is found using Eqs. (119) and (120). The phase difference is zero for the symmetric mode at a frequency of $\omega_{2}$ and the phase difference is $\pi$ at the frequency $\omega_{2}$ for the antisymmetric mode. The remaining parameters are $\bar{s}=6, T_{0}=0.43$, and $\omega_{0}=450016$.

where

$$
\begin{aligned}
& \phi_{1}=\tan ^{-1}\left(\frac{\operatorname{Re}\left(\hat{\bar{x}}_{1}\left(\tilde{\omega}_{d}\right)\right)}{\operatorname{Im}\left(\hat{\bar{x}}_{1}\left(\tilde{\omega}_{d}\right)\right)}\right), \\
& \phi_{2}=\tan ^{-1}\left(\frac{\operatorname{Re}\left(\hat{\bar{x}}_{2}\left(\tilde{\omega}_{d}\right)\right)}{\operatorname{Im}\left(\hat{\bar{x}}_{2}\left(\tilde{\omega}_{d}\right)\right)}\right),
\end{aligned}
$$

where $\tilde{\omega}_{d}=\omega_{d} / \omega_{0}, \operatorname{Re}$ is the real part, and Im is the imaginary part. The phase difference between the two cylinders is

$$
\Delta \phi=\phi_{1}-\phi_{2} .
$$

The normalized phase difference is plotted in Fig. 13 as a function of the driving frequency $\omega_{d}$. For $\omega_{d} \rightarrow 0$ the phase difference becomes $\Delta \phi=\pi / 4$. For $\omega_{d}=\omega_{2}$ the phase difference is $\Delta \phi=0$, indicating that this is the symmetric mode solution. For $\omega_{d}=\omega_{1}$ the phase difference is $\Delta \phi=\pi$, indicating the antisymmetric mode solution. Finally, for $\omega_{d} \rightarrow$ $\infty$ the phase difference $\Delta \phi$ approaches zero.

In the limit of $\beta_{0} \rightarrow 0$ the viscous boundary layer grows to infinite extent. In two-dimensional fluid dynamics, as we are using here, this leads to the well-known Stokes paradox, where it is not possible to satisfy both the no-slip boundary condition at the cylinder surface and the far-field flow condition (cf. Ref. [29]). This leads to the Oseen correction, which is precisely what was used when studying the fluid-coupled motion of micron-scale beads in the viscous limit [17]. Our equations are not valid in the limit of $\beta_{0} \rightarrow 0$; however, they are valid in the limit of $\beta_{0} \ll 1$, which contains the range of interest for nanoscale oscillators of interest that are dominated by viscous forces. A similar analysis to that of Ref. [17] could be conducted that would be valid for $\beta_{0} \rightarrow 0$ but is not included here.

Figure 14 illustrates the cylinder dynamics for the case of very viscous interactions. The solid curves illustrate the variation of the amplitude with frequency for three orders of magnitude of the frequency parameter where $\beta_{0} \leqslant 1$. Figure 14(a) illustrates the dynamics of cylinder 1 and Fig. 14(b) illustrates the dynamics for cylinder 2 . The presence of viscous damping results in the resonances having finite 

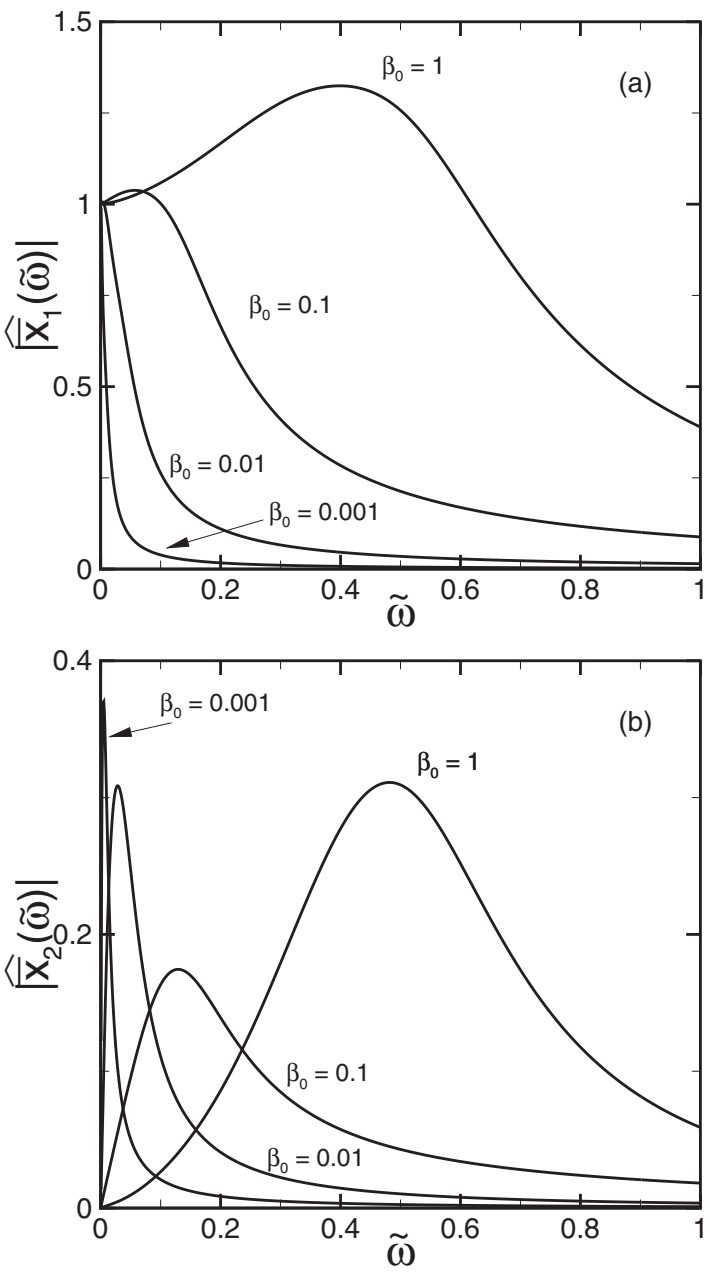

FIG. 14. The amplitude frequency plot for $\left|\hat{\bar{x}}_{1}(\tilde{\omega})\right|$ in panel (a) and $\left|\hat{\bar{x}}_{2}(\tilde{\omega})\right|$ in panel (b) for two cylinders with strong viscous interactions. The different curves are for different values of $\beta_{0}$ where $\beta_{0}=1,0.1,0.01,0.001$, where the amplitude of the peak decreases with decreasing $\beta_{0}$ and moves toward lower frequency. The curves are found by evaluating Eqs. (90) and (91). The remaining parameters used are $\bar{s}=6$ and $T_{0}=0.43$.

amplitude, in broadening the peak, and in shifting the response to lower frequencies, as expected. It is also clear from the plots that the mode splitting has been damped out and the dynamics are either overdamped or best described by a single peak.

In light of these results, the mode splitting is due entirely to the potential component of the fluid flow field. The oscillation of a cylinder causes an additional mass loading term that depends on the acceleration of the cylinder. The oscillation of a neighboring cylinder causes an additional mass loading term that depends on the acceleration of the neighbor. This can be seen on the right-hand side of Eqs. (105) and (106). Therefore, the important physics is the summing or differencing of these additional mass loading terms multiplied by the acceleration of the respective cylinder. The summing or differencing will depend on the relative phase difference between the two cylinders, as shown in Fig. 5. The two normal modes occur at frequencies $\omega_{1}$ and $\omega_{2}$, where $\omega_{1}>\omega_{2}$. At $\omega_{1}$ the two cylinders are out of phase and the potential flow field from each cylinder is adding to the motion of each cylinder. This results in the amplitude of oscillation being larger at $\omega_{1}$. At $\omega_{2}$ the two cylinders oscillate in phase with each other. In this case, the potential flow field from each cylinder opposes the motion of its neighbor which results in a smaller amplitude of oscillation. For a frequency between $\omega_{1}$ and $\omega_{2}$, the potential interactions cancel and the motion of the cylinders is significantly reduced. The separation of the peaks of the two modes $\Delta \tilde{\omega}$ depends on $T_{0}$ and $\bar{s}$. The value of $\Delta \tilde{\omega}$ goes to zero as $\bar{s}$ becomes large or as $T_{0}$ goes to zero.

\section{The fluid-coupled motion of two cylinders when driven by Brownian motion}

We now discuss the stochastic dynamics of a pair of cylinders immersed in a viscous fluid that are driven by Brownian
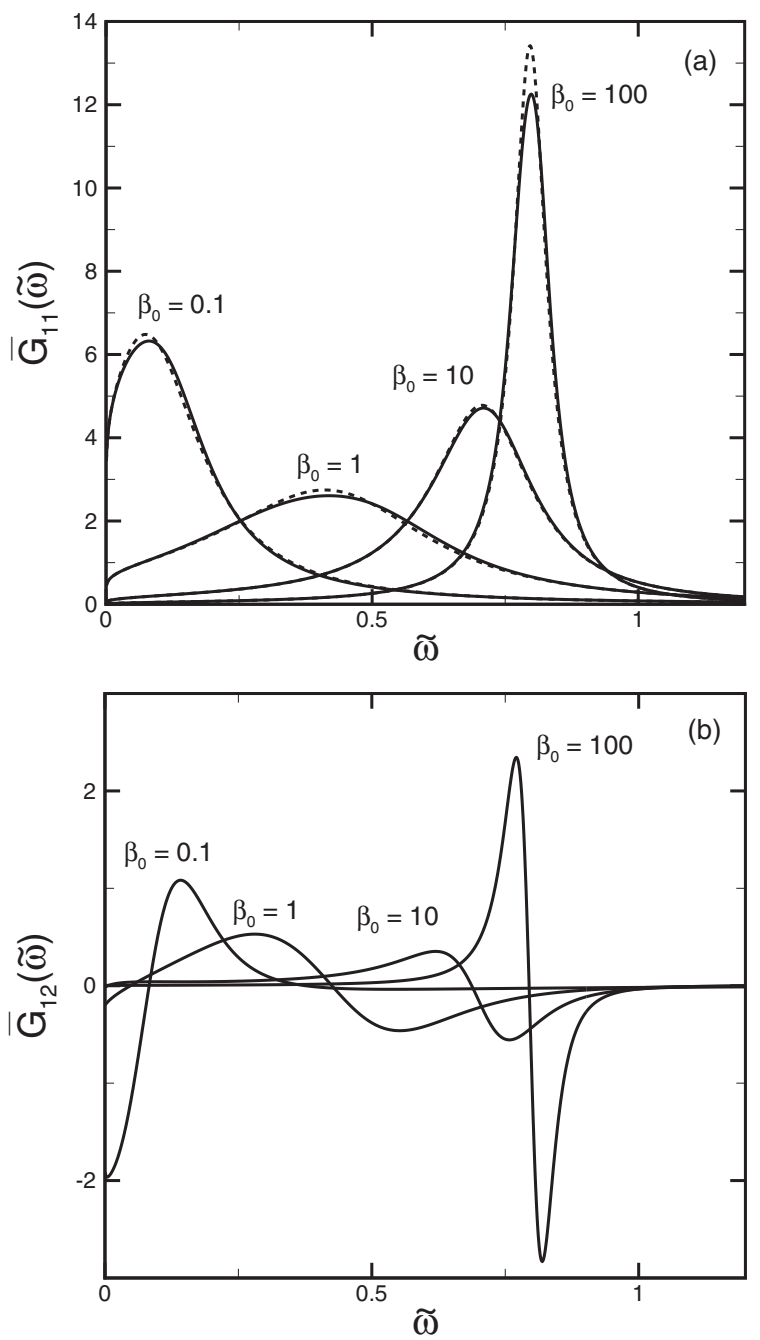

FIG. 15. The variation of the noise spectra with frequency parameter for the stochastic motion of a cylinder pair driven by Brownian motion. The cylinder separation is $\bar{s}=6$, the mass loading parameter is $T_{0}=0.43$, and the frequency parameter varies over the range $0.1 \leqslant \beta_{0} \leqslant 100$. (a) The variation of the noise spectra $\bar{G}_{11}$. The solid lines are the predictions given by Eq. (99) and the dashed lines are the predictions for the dynamics of a single cylinder in the absence of a neighboring cylinder. (b) The variation of the noise spectra for the correlated motion $\bar{G}_{12}$. The solid lines are the predictions found by evaluating Eq. (100). 
motion. The variation of the normalized noise spectra with the frequency parameter are shown in Fig. 15. In this figure the mass loading parameter is $T_{0}=0.43$ and the cylinder separation is $\bar{s}=6$. Figure 15 (a) illustrates the variation of $\bar{G}_{11}(\tilde{\omega})$, where the dashed lines are the noise spectra in the absence of a neighboring cylinder and the solid lines are the noise spectra with a neighboring cylinder given by Eq. (99). The general trend in the dynamics is similar to what was found for the driven dynamics as shown in Fig. 10(a). Over this range of frequency parameters the noise spectra $\bar{G}_{11}$ are not affected significantly by the presence of the neighboring cylinder. As the frequency parameter is reduced the peak in the noise spectrum becomes wider and moves towards lower frequencies. These dynamics are well represented by a single peak.
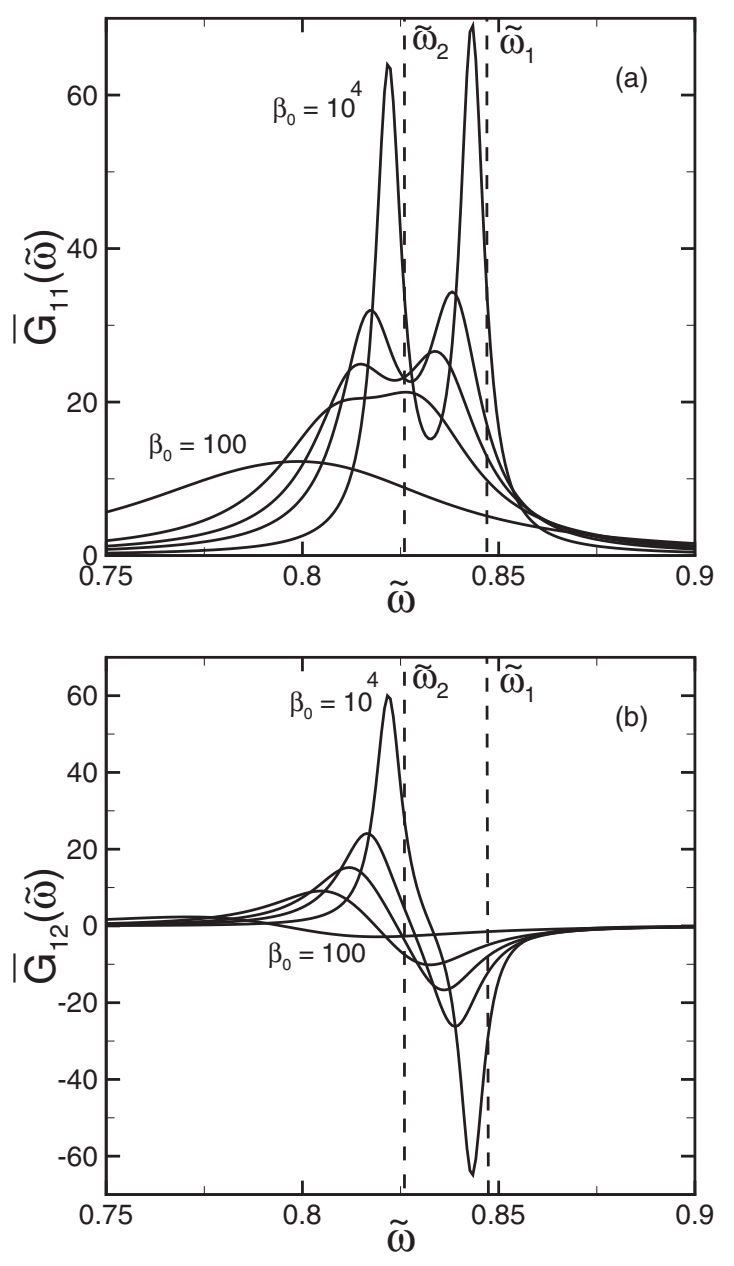

FIG. 16. The variation of the noise spectra with frequency parameter for the stochastic motion of a cylinder pair driven by Brownian motion. The curves shown are for large values of $\beta_{0}$ where $\beta_{0}=100,500,1000,2000,10^{4}$. The curves for $\beta_{0}=100,10^{4}$ are labeled with the remaining curves appearing in sequence. The resonant frequencies of the two normal mode solutions $\tilde{\omega}_{1}$ and $\tilde{\omega}_{2}$ are indicated by the dashed lines. The cylinder separation is $\bar{s}=6$, and the mass loading parameter is $T_{0}=0.43$. (a) The variation of the noise spectra $\bar{G}_{11}$; the solid lines are the predictions given by Eq. (99). (b) The variation of the noise spectra for the correlated motion $\bar{G}_{12}$; the solid lines are the predictions found by evaluating Eq. (100).
The noise spectra for the correlated motion of a pair of cylinders driven by Brownian motion $\bar{G}_{12}$ are shown in Fig. 15(b). The solid lines are $\bar{G}_{12}$ using Eq. (100). The noise spectra $\bar{G}_{12}$ have both positive and negative peaks. The magnitude of $\bar{G}_{12}$ vanishes at the frequency where there is a peak $\bar{G}_{11}$. For $\beta_{0} \gtrsim 1$ the noise spectrum contains two lobes with a positive lobe followed by a negative lobe. This is similar to what has been observed for a pair of closely spaced micron-scale atomic force microscopy cantilevers in fluid where $\beta_{0} \approx 100$ [21,22]. As the frequency parameter is reduced the dynamics shift to lower frequencies with the addition of a negative lobe in the correlated noise spectrum at low frequencies. These results are similar to what has been computed for a pair of nanoscale cantilevers in fluid where $\beta_{0} \approx 0.1[19,21]$.

It is interesting to note that the structure of the noise spectrum changes as the frequency parameter is increased. This is illustrated in Fig. 16 for a cylinder pair over a large range of frequency parameters where $100 \leqslant \beta_{0} \leqslant 10^{4}$. Figure 16(a) illustrates the noise spectrum $\bar{G}_{11}$ which shows the mode splitting that occurs for large values of the frequency parameter. For $\beta_{0} \gtrsim 10^{3}$ the noise spectrum contains two peaks where the magnitude of the lower frequency peak is smaller than the magnitude of the higher-frequency peak. These two peaks can be identified as the two normal modes from an analysis including the potential fluid interactions. For reference, the resonant frequencies of the two normal modes from the potential interaction analysis are indicated by the dashed lines. We point out that it is not possible to conduct an analysis only containing the potential interactions for the stochastic motion as this requires the presence of dissipation as indicated by the fluctuation-dissipation theorem. In Fig. 16(a) the antisymmetric mode is the larger peak at higher frequency and the symmetric mode is the smaller peak at lower frequency. The noise spectrum of the correlated motion is shown in Fig. 16(b). The noise spectra contain a positive and negative lobe for the range of frequency parameter that is shown, which is a signature of the mode splitting present.

\section{CONCLUSIONS}

We anticipate that our results will provide useful physical insights into the correlated dynamics of small elastic objects in a viscous fluid for a wide range of conditions. These insights will be useful in the development of new technologies that exploit these fluid-coupled dynamics. Our approach is quite general and a number of extensions are readily available. Although we used a two-dimensional cylindrical geometry for the oscillating objects, one could implement the available expressions for oscillating thin blades [30], spheres [23], or rectangular geometries [30]. Our approach could also be used to explore the dynamics of higher modes of oscillations using the hydrodynamic function given in Refs. [31,32]. Overall, for fluid-solid interaction problems in the regime described by the unsteady Stokes equations, we have elucidated the subtle interaction between the viscous and potential contributions to the flow field. It would be interesting to compare the results presented here with experimental measurement over a wide range of conditions and cylinder separations. 


\section{ACKNOWLEDGMENTS}

We acknowledge support from AFOSR under Grant No. FA9550-07-1-0222 (M.R.P. and M.T.C.) and a Virginia Tech
ASPIRES grant (M.R.P.). We also acknowledge many useful interactions with Michael Roukes, Zhipeng Zheng, and Carlos Carvajal.
[1] K. L. Ekinci, V. Yakhot, S. Rajauria, C. E. Colosqui, and D. M. Karabacak, Lab on a Chip 10, 3013 (2010).

[2] T. M. Squires and T. Mason, Annu. Rev. Fluid Mech. 24, 413 (2010).

[3] R. García and R. Pérez, Surf. Sci. Rep. 47, 197 (2002).

[4] A. Raman, J. Melcher, and R. Tung, Nanotoday 3, 20 (2008).

[5] J. L. Arlett, M. R. Paul, J. Solomon, M. C. Cross, S. E. Fraser, and M. L. Roukes, Lect. Notes Phys. 711, 241 (2007).

[6] J. L. Arlett, E. B. Myers, and M. L. Roukes, Nat. Nanotechnol. 6, 203 (2011).

[7] F. M. Battiston, J.-P. Ramseyer, H. P. Lang, M. K. Baller, C. Gerber, J. K. Gimzewski, E. Meyer, and H.-J. Guntherodt, Sensors and Actuators B 77, 122 (2001).

[8] R. E. Goldstein, M. Polin, and I. Tuval, Phys. Rev. Lett. 103, 168103 (2009).

[9] A. Vilfan and F. Julicher, Phys. Rev. Lett. 96, 058102 (2006).

[10] J. C. Meiners and S. R. Quake, Phys. Rev. Lett. 84, 5014 (2000).

[11] M. Radiom, B. A. Robbins, C. D. F. Honig, W. J. W., M. R. Paul, and W. A. Ducker, Rev. Sci. Instrum. 83, 043908 (2012).

[12] E. Lauga and T. R. Powers, Rep. Prog. Phys. 72, 096601 (2009).

[13] W. Han, S. M. Lindsay, and T. Jing, Appl. Phys. Lett. 69, 411 (1996).

[14] I. Bargatin, I. Kozinsky, and M. L. Roukes, Appl. Phys. Lett. 90, 093116 (2007)

[15] T. E. Schäffer, J. P. Cleveland, D. Ohnesorge, D. Walters, and P. Hansma, J. Appl. Phys. 80, 3622 (1996).

[16] Y. Levin, Phys. Rev. D 57, 659 (1998).
[17] J. C. Meiners and S. R. Quake, Phys. Rev. Lett. 82, 2211 (1999).

[18] S. Basak and A. Raman, Phys. Fluids 19, 017105 (2007).

[19] M. R. Paul and M. C. Cross, Phys. Rev. Lett. 92, 235501 (2004).

[20] M. R. Paul, M. T. Clark, and M. C. Cross, Nanotechnology 17, 4502 (2006).

[21] M. T. Clark and M. R. Paul, Int. J. Nonlin. Mech. 42, 690 (2007).

[22] C. D. F. Honig, M. Radiom, B. A. Robbins, W. J. W., M. R. Paul, and W. A. Ducker, Appl. Phys. Lett. 100, 053121 (2012).

[23] G. G. Stokes, Camb. Phil. Soc. Trans. 9, 8 (1851).

[24] L. Rosenhead, Laminar Boundary Layers (Oxford University Press, Oxford, 1963).

[25] C. Pozrikidis, Boundary Integral and Singularity Methods for Linearized Viscous Flow (Cambridge University Press, Cambridge, 1992).

[26] M. R. Paul and J. E. Solomon, Nanodevices for Life Sciences (Wiley-VCH, Weinheim, 2005), Vol. 4.

[27] S. Kim and S. Karrila, Microhydrodynamics Principles and Selected Applications (Dover, London, 2005).

[28] All of our numerical simulations were done using the finiteelement solver available from ADINA R \& D, Inc., 71 Elton Avenue, Watertown, MA 02472 (2013).

[29] G. K. Batchelor, An Introduction to Fluid Dynamics (Cambridge University Press, New York, 1967).

[30] J. E. Sader, J. Appl. Phys. 84, 64 (1998).

[31] C. Van Eysden and J. E. Sader, J. Appl. Phys. 101, 044908 (2007).

[32] C. A. Van Eysden and J. E. Sader, J. Appl. Phys. 106, 094904 (2009). 